\title{
Decay-Assisted Laser Spectroscopy of Neutron-Deficient Francium
}

K. M. Lynch, ${ }^{1,2,3, *}$ J. Billowes, ${ }^{1}$ M. L. Bissell, ${ }^{3}$ I. Budinčević, ${ }^{3}$ T. E. Cocolios, ${ }^{1,2}$ R. P. De Groote, ${ }^{3}$ S. De Schepper, ${ }^{3}$ V. N. Fedosseev, ${ }^{4}$ K. T. Flanagan, ${ }^{1}$ S. Franchoo, ${ }^{5}$ R. F. Garcia Ruiz, ${ }^{3}$ H. Heylen, ${ }^{3}$ B. A. Marsh, ${ }^{4}$ G. Neyens, ${ }^{3}$ T. J. Procter, ${ }^{1, \dagger}$ R. E. Rossel, ${ }^{4,6}$ S. Rothe, ${ }^{4,6}$ I. Strashnov, ${ }^{1}$ H. H. Stroke, ${ }^{7}$ and K. D. A. Wendt ${ }^{6}$

${ }^{1}$ School of Physics and Astronomy, The University of Manchester, Manchester M13 9PL, United Kingdom

${ }^{2}$ ISOLDE, PH Department, CERN, CH-1211 Geneva-23, Switzerland

${ }^{3}$ Instituut voor Kern-en Stralingsfysica, KU Leuven, B-3001 Leuven, Belgium

${ }^{4}$ EN Department, CERN, CH-1211 Geneva 23, Switzerland

${ }^{5}$ Institut de Physique Nucléaire d'Orsay, F-91406 Orsay, France

${ }^{6}$ Institut für Physik, Johannes Gutenberg-Universität Mainz, D-55128 Mainz, Germany

${ }^{7}$ Department of Physics, New York University, New York, New York 10003, USA

(Received 6 January 2014; published 28 March 2014)

This paper reports on the hyperfine-structure and radioactive-decay studies of the neutron-deficient francium isotopes ${ }^{202-206} \mathrm{Fr}$ performed with the Collinear Resonance Ionization Spectroscopy (CRIS) experiment at the ISOLDE facility, CERN. The high resolution innate to collinear laser spectroscopy is combined with the high efficiency of ion detection to provide a highly sensitive technique to probe the hyperfine structure of exotic isotopes. The technique of decay-assisted laser spectroscopy is presented, whereby the isomeric ion beam is deflected to a decay-spectroscopy station for alpha-decay tagging of the hyperfine components. Here, we present the first hyperfine-structure measurements of the neutron-deficient francium isotopes ${ }^{202-206} \mathrm{Fr}$, in addition to the identification of the low-lying states of ${ }^{202,204} \mathrm{Fr}$ performed at the CRIS experiment.

DOI: 10.1103/PhysRevX.4.011055

\section{INTRODUCTION}

Recent advances in high-resolution laser spectroscopy have resulted in the ability to measure short-lived isotopes with yields of less than 100 atoms/s [1,2]. The Collinear Resonance Ionization Spectroscopy (CRIS) experiment [3], located at the ISOLDE facility, CERN, aims to push the limits of laser spectroscopy further, performing hyperfine-structure measurements on isotopes at the edges of the nuclear landscape. It provides a combination of highdetection efficiency, high resolution, and ultralow background, allowing measurements to be performed on isotopes with yields below, in principle, 1 atom/s.

The first optical measurements of francium were performed in 1978. Liberman et al. identified the $7 s^{2} S_{1 / 2} \rightarrow$ $7 p^{2} P_{3 / 2}$ atomic transition, performing hyperfine-structure and isotope-shift measurements first with low-resolution [4] and later with high-resolution laser spectroscopy [5]. The wavelength of this transition $\lambda(D 2)=717.97(1) \mathrm{nm}$ was in excellent agreement with the prediction of Yagoda

\footnotetext{
*kara.marie.lynch@cern.ch

†Present address: TRIUMF, Vancouver, British Columbia V6T 2A3, Canada.

Published by the American Physical Society under the terms of the Creative Commons Attribution 3.0 License. Further distribution of this work must maintain attribution to the author(s) and the published article's title, journal citation, and DOI.
}

Subject Areas: Nuclear Physics

[6], made in 1932 before francium was discovered. Further measurements of francium followed in the next decade. High-resolution optical measurements were performed on both the $7 s^{2} S_{1 / 2} \rightarrow 7 p^{2} P_{3 / 2}$ atomic transition [7-9] and the $7 s^{2} S_{1 / 2} \rightarrow 8 p^{2} P_{3 / 2}$ transition [10], along with transitions into high-lying Rydberg states [11]. The CRIS technique, a combination of collinear laser spectroscopy and resonance ionization, was originally proposed by Kudriavtsev and Letokhov in 1982 [12], but the on-line experimental realization of the technique was not performed until 1991 on ytterbium atoms [13].

The ability to study the neutron-deficient francium $(Z=87)$ isotopes at the CRIS beam line offers the unique opportunity to answer questions arising from the study of the nuclear structure in this region of the nuclear chart. As the isotopes above the $Z=82$ shell closure become more neutron deficient, a decrease in the excitation energy of the $\left(\pi 1 i_{13 / 2}\right)_{13 / 2^{+}},\left(\pi 2 f_{7 / 2}\right)_{7 / 2^{+}},\left(\nu 1 i_{13 / 2}\right)_{13 / 2^{+}}$, and $\left(\pi 3 s_{1 / 2}^{-1}\right)_{1 / 2^{+}}$states is observed. In ${ }^{185} \mathrm{Bi}(Z=83)$ and ${ }^{195} \mathrm{At}(Z=85)$, the $\left(\pi 3 s_{1 / 2}^{-1}\right)_{1 / 2^{+}}$deformed-intruder state has been observed to be the ground state $[14,15]$.

Recent radioactive-decay measurements suggest the existence of a $\left(\pi 3 s_{1 / 2}^{-1}\right)_{1 / 2^{+}}$proton-intruder state for ${ }^{203} \mathrm{Fr}$ and, with a lower excitation energy, for ${ }^{201} \mathrm{Fr}$, suggesting that this state may become the ground state in ${ }^{199} \operatorname{Fr}[16,17]$. The intruder configurations polarize the nucleus, creating significant deformation. From the study of the nuclear structure of the neutron-deficient francium isotopes toward 


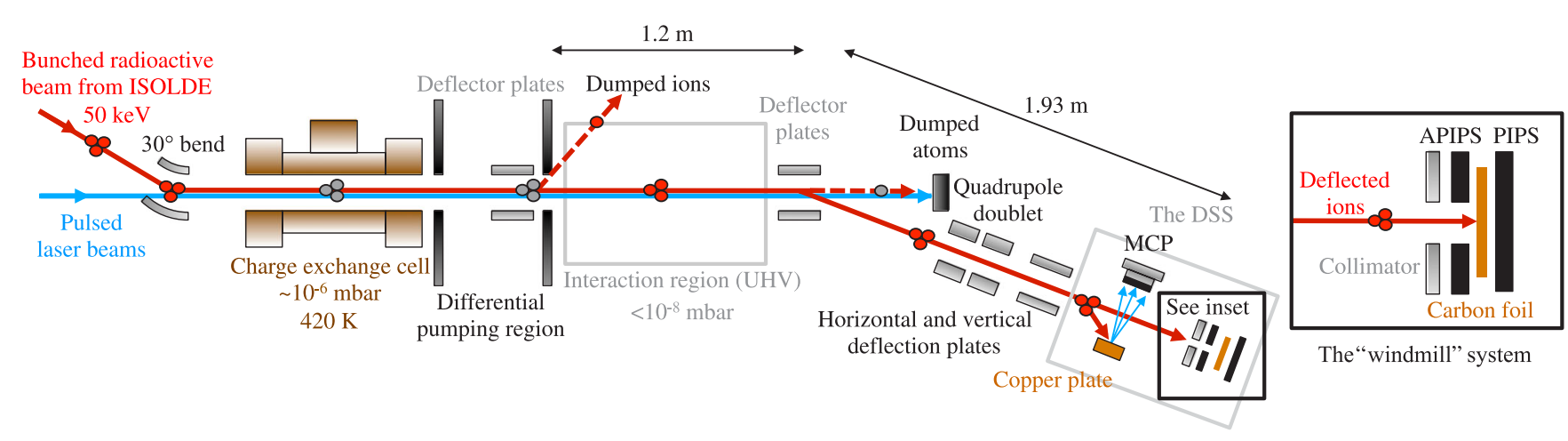

FIG. 1. Schematic diagram of the CRIS beam line. Laser ions can be deflected to a copper plate, and the corresponding secondary electrons can be detected by the MCP or implanted into a carbon foil for alpha-decay spectroscopy. Inset: The DSS "windmill" system for alpha-decay tagging.

${ }^{199} \mathrm{Fr}$ (by measuring the magnetic dipole moments and change in mean-square charge radii of the ground and isomeric states), the quantum configuration of the states and the shape of the nuclei can be investigated.

Radioactive-decay measurements on the neutrondeficient francium isotopes have aimed to determine the level structure of the low-lying nuclear states, but their exact nature is still unknown [16-19]. High-resolution collinear laser spectroscopy has allowed determination of the ground-state properties of ${ }^{204,205,206} \mathrm{Fr}$ [20], confirming the tentative spin assignments. The spin of ${ }^{205} \mathrm{Fr}$ was measured to be $9 / 2^{-}$, the ground-state spins of ${ }^{204,206} \mathrm{Fr}$ were confirmed as $3^{(+)}$, but the low-lying spin $\left(7^{+}\right)$and $\left(10^{-}\right)$isomers are still under investigation.

General methods of isomer identification have already been achieved with in-source laser spectroscopy (see Ref. [21] and references therein). In the case of ${ }^{68,70} \mathrm{Cu}$ [22], following the selection of isomeric beams, experiments such as Coulomb excitation [23] and mass measurements [24] have been performed. However, these experiments suffered from isobaric contamination, as well as significant ground-state contamination due to the Doppler broadening of the hyperfine resonances of each isomer [1]. One way of addressing the difficulties of in-source laser spectroscopy (isobaric contamination, Doppler broadening, pressure broadening) is selecting the ground or isomeric state of interest by resonance ionization in a collinear geometry.

In a sub-Doppler geometry, the process of isomerselective resonance laser ionization [21] can result in a high-purity isomeric beam. Deflection of the pure-state ion beam to the decay-spectroscopy station allows identification of the hyperfine component with alpha-decay spectroscopy.

\section{EXPERIMENTAL TECHNIQUE}

Radioactive ion beams of francium are produced at the ISOLDE facility, CERN [25], by impinging $1.4-\mathrm{GeV}$ protons onto a thick uranium carbide $\left(\mathrm{UC}_{x}\right)$ target (up to $2-\mu \mathrm{A}$ integrated proton current). The radioisotopes are surface ionized through interaction with the rhenium coating on the hot (2400-K) tantalum transfer line and extracted from the target-ion source at $50 \mathrm{keV}$. The isotope of interest is mass selected using the high-resolution separator and bunched (at $31.25 \mathrm{~Hz}$ ) with the radio-frequency ISOLDE cooler (ISCOOL) [26,27]. The bunched-ion beam is deflected into the CRIS beam line and transported through a potassium-vapor charge-exchange cell $(420-\mathrm{K}$, approximately $10^{-6}$-mbar chamber pressure, $6 \times 10^{-4}$-mbar vapor pressure [28]) to be neutralized. In the 1.2-m-long interaction region, the arrival of the atomic bunch is synchronized with two copropagating pulsed laser beams to excite the state of interest followed by ionization in a stepwise scheme. The temporal length of the atomic bunch is $2-3 \mu \mathrm{s}$, corresponding to a spatial length of $45-70 \mathrm{~cm}$. To reduce the background signal resulting from nonresonant collisional ionization, the interaction region aims at ultrahighvacuum (UHV) conditions. A pressure of $<10^{-8} \mathrm{~m}$ bar is achieved during this experiment. A schematic diagram of the CRIS beam line is shown in Fig. 1.

\section{A. Collinear resonance ionization spectroscopy}

The resonant-excitation step from the $7 s^{2} S_{1 / 2}$ electronic ground state to the $8 p^{2} P_{3 / 2}$ state is probed with $422.7-\mathrm{nm}$ light. The laser light of this resonant step is provided by a narrow-band Ti:sapphire laser of the resonance ionization laser ion source (RILIS) installation [29,30], pumped by the second harmonic output of a neodymium-doped yttrium aluminum garnet (Nd:YAG) laser (model: Photonics Industries DM-60-532, $10 \mathrm{kHz}$ ). The fundamental output from the tunable Ti:sapphire laser is frequency doubled using a barium borate crystal to produce the required 422.7$\mathrm{nm}$ laser light. The light is fiber coupled into the CRIS beam line through $35 \mathrm{~m}$ of multimode optical fiber (approximately 100-mW output). The laser linewidth of $1.5 \mathrm{GHz}$ limits the resolution achieved in the present experiment, allowing only the lower-state $\left(7 s^{2} S_{1 / 2}\right)$ splitting to be fully resolved. The second (nonresonant) transition from the $8 p^{2} P_{3 / 2}$ state to the continuum is 
driven using 1064-nm light. This light is produced by a fundamental Nd:YAG laser (model: Quanta-Ray LAB 130, operated at $31.25 \mathrm{~Hz}$ ) next to the CRIS setup, temporally overlapped with the 422.7-nm laser beam and aligned through the laser-atom interaction region. The standard repetition rate of the RILIS lasers $(10 \mathrm{kHz})$ limits the repetition rate of the 1064-nm laser light to $31.25 \mathrm{~Hz}$. (One out of every 320 pulses of 422.7-nm laser light is utilized.) The bunching of the ion beam with ISCOOL is matched to the lower repetition rate of $31.25 \mathrm{~Hz}$ to overlap the atom bunch with the two laser pulses every $32 \mathrm{~ms}$.

The synchronization of the first- and second-step laser pulses and the release of the ion bunch from ISCOOL are controlled by a Quantum Composers digital-delay generator (model: QC9258). The 10-kHz pulse generator of the Ti:sapphire pump laser acts as the master clock, triggering the delay generator to output a sequence of transistortransistor logic pulses to synchronize the 1064-nm laser light and the ion bunch with the 422.7-nm light, allowing resonance ionization of the francium atoms to occur. The laser ions are detected by a microchannel plate (MCP) housed in the decay-spectroscopy station (DSS). The electronic signal from the MCP is digitized by a LeCroy oscilloscope (model: WavePro 725Zi, 2-GHz bandwidth, 8-bit analogue-to-digital converter, 20 giga-samples/s), triggered by the digital-delay generator. The data are transferred from the oscilloscope using a LabVIEW program.

The frequency of the resonant-excitation step, the 422.7-nm laser light, is scanned to study the $7 s^{2} S_{1 / 2} \rightarrow$ $8 p^{2} P_{3 / 2}$ atomic transition. The scanning and stabilization of the frequency are controlled by the RILIS Equipment Acquisition and Control Tool (REACT), a LabVIEW control program package that allows for remote control, equipment monitoring, and data acquisition [31]. The scanning is achieved by controlling the etalon tilt angle inside the Ti:sapphire laser resonator to adjust the laser wavelength, which is measured with a HighFinesse wave meter (model: WS7) and calibrated with a frequencystabilized HeNe laser. The francium experimental campaign at CRIS marks the first implementation of the REACT framework for external users. The remote-control LabVIEW interface for the Ti:sapphire laser runs locally at the CRIS setup, allowing independent laser scanning and control.

\section{B. Decay-assisted laser spectroscopy}

The technique of decay-assisted collinear laser spectroscopy is further developed at the CRIS beam line to take advantage of the ultrapure ion beams produced by resonance ionization in a collinear geometry [32]. The selectivity from resonance ionization of an isotope is a result of the selectivity of the Lorentzian profile of the natural linewidth (approximately $12.5 \mathrm{MHz}$ ) of the state and the Gaussian profile of the laser linewidth (approximately $1.5 \mathrm{GHz}$ ). At a frequency separation of $4 \mathrm{GHz}$, the
Gaussian component falls to $1 \%$ of its peak intensity and the selectivity is dominated by the natural linewidth of the state. Thus, the maximum selectivity from resonance ionization is given by Eq. (1):

$$
S=\prod_{n=1}^{N}\left(\frac{\Delta \omega_{A B, n}}{\Gamma_{n}}\right)^{2}=\prod_{n=1}^{N} S_{n},
$$

where $\Delta \omega_{A B}$ is the separation in frequency of the two states $(A$ and $B), \Gamma_{n}$ is the FWHM of the natural linewidth of the state, $S_{n}$ is the selectivity of the transition, and $N$ is the number of transitions used. The total selectivity of a resonance ionization process is given by the product of the individual selectivities. In the case of the two states being the ground state and isomer, the selectivity can be calculated from Eq. (1). When the two states are the isotope of interest and contamination from a neighboring isotope, additional selectivity can be gained from the kinematic shift since the laser is overlapped with an accelerated beam.

In addition to hyperfine-structure studies with ion detection, the decay-spectroscopy station can be used to identify the hyperfine components of overlapping structures. The DSS allows the hyperfine structure of two states to be separated by exploiting their characteristic radioactivedecay mechanisms. The separation results in a smaller error associated with the hyperfine parameters and a better determination of the extracted nuclear observables.

The DSS consists of a rotatable-wheel implantation system [33]. It is based on the design from KU Leuven [34] (Fig. 1 of Ref. [35]), which has provided results in a number of successful experiments (see Ref. [36] and references therein). The wheel holds nine carbon foils, produced at the GSI target laboratory [37], with a thickness of 20 (1) $\mu \mathrm{g} \mathrm{cm}^{-2}$ (approximately $90 \mathrm{~nm}$ ) into which the ion beam is implanted (at a depth of approximately $25 \mathrm{~nm}$ ).

Two Canberra passivated implanted planar silicon (PIPS) detectors for charged-particle detection (e.g., alpha, electron, fission fragments) are situated on either side of the implantation carbon foil, as shown in Fig. 1. One PIPS detector (model: BKA 300-17 AM, thickness $300 \mu \mathrm{m}$ ) sits behind the carbon foil and another annular PIPS (APIPS) (model: BKANPD 300-18 RM, thickness $300 \mu \mathrm{m}$, with an aperture of $4 \mathrm{~mm}$ ) is placed in front of the carbon foil. The detectors are connected to charge-sensitive Canberra preamplifiers (model: 2003BT) via a UHV type-C subminiature electrical feedthrough.

Laser-produced ions from the interaction region in the CRIS beam line are deflected to the DSS by applying a potential difference between a pair of vertical electrostatic plates; see Fig. 1. The deflected ion beam is implanted into the carbon foil, after passing though a collimator with a 4-mm aperture and the APIPS detector. The collimator shields the APIPS detector from direct implantation of radioactive ions into the silicon wafer; see Fig. 1. Decay products from the carbon foil can be measured by either the 
APIPS or PIPS detector, with a total solid-angle coverage of $63 \%$ (simulated, assuming a uniform distribution of implanted activity). Operation of the single APIPS detector during the experiment gives an alpha-detection efficiency of $25 \%$. An electrical contact is made to the collimator, allowing the current generated by the ion beam when it strikes the collimator to be measured and the plate to be used as a beam-monitoring device. When it is not in use, it is electrically grounded to avoid charge buildup. A Faraday cup is installed in the location of one of the carbon foils. This copper plate (thickness $0.5 \mathrm{~mm}$, diameter $10 \mathrm{~mm}$ ) is electrically isolated from the steel wheel by polyether ether ketone (PEEK) rings and connected by a spot-welded Kapton cable attached to a rotatable Bayonet NeillConcelman (BNC) connection in the center of the wheel [33].

The alpha-decay-spectroscopy data are acquired with a digital data-acquisition system, consisting of XIA digital gamma-finder (DGF) revision- $D$ modules [38]. Each module has four input channels with a $40-\mathrm{MHz}$ sampling rate. Signals fed into the digital data-acquisition system are self-triggered with no implementation of master triggers.

Because of the reflective surface of the inside of the vacuum chambers, a significant fraction of 1064-nm laser light is able to scatter into the silicon detectors. Despite the collimator in front of the APIPS detector to protect it from ion implantation (and laser light), the infrared light causes a shift in the baseline of the signal from the silicon detector. The resulting shift requires the parameters for the DGF modules to be adjusted to account for this effect on-line, since the reflections are due to the particular setup of the experiment (power and laser-beam path).

The low energy resolution of the APIPS detector is associated with the necessity of optimizing the DGF parameters on-line with the radioactive ${ }^{221} \mathrm{Fr}\left[t_{1 / 2}=4.9(2) \mathrm{min}\right]$. In addition, a fluctuating baseline resulting from the changing power of the 1064-nm laser light means that only a resolution of $30 \mathrm{keV}$ at $6.341 \mathrm{MeV}$ is achieved. The energy resolution, however, is sufficient to identify the characteristic alpha decays of the neutron-deficient francium isotopes under investigation.

\section{RESULTS}

The hyperfine structures of the neutron-deficient francium isotopes ${ }^{202-206} \mathrm{Fr}$ are measured with collinear resonance ionization spectroscopy, with respect to the reference isotope ${ }^{221} \mathrm{Fr}$. This paper follows the recent publication reporting the hyperfine-structure studies of $202,203,205 \mathrm{Fr}$ [39]. During the experimental campaign, the neutron-rich francium isotopes ${ }^{218 m, 219,229,231} \mathrm{Fr}$ are also studied. A detailed description of the nature of these isotopes will be the topic of a future publication [40].

The resonance spectrum of the $7 s^{2} S_{1 / 2} \rightarrow 8 p^{2} P_{3 / 2}$ transition is fit with a $\chi^{2}$-minimization routine. The hyperfine $A_{P_{3 / 2}}$ factor is fixed to the ratio of the $7 s^{2} S_{1 / 2} \rightarrow$ $8 p^{2} P_{3 / 2}$ transition of $A_{P_{3 / 2}} / A_{S_{1 / 2}}=+22.4 / 6209.9$, given in the literature [10]. For the $8 p^{2} P_{3 / 2}$ state, the hyperfine $B_{P_{3 / 2}}$ factor is small enough to have no impact on the fit to the data and is consequently set to zero [41].

The intensities of the hyperfine transitions $S_{F F^{\prime}}$ between hyperfine levels $F$ and $F^{\prime}$ (with angular momenta $J$ and $J^{\prime}$, respectively) are related to the intensity of the underlying fine-structure transition $S_{J J^{\prime}}$ [2]. The relative intensities of the hyperfine transitions are given by

$$
\frac{S_{F F^{\prime}}}{S_{J J^{\prime}}}=(2 F+1)\left(2 F^{\prime}+1\right)\left\{\begin{array}{lll}
F & F^{\prime} & 1 \\
J^{\prime} & J & I
\end{array}\right\}^{2}
$$

where the curly brackets denote the Wigner 6- $j$ coefficient. Although these theoretical intensities are only strictly valid for closed two-level systems, and there is jitter on the temporal overlap of the two laser pulses in the interaction region, they are used as the currently most reliable estimate.

The $A_{S_{1 / 2}}$ factor and the centroid frequency of the hyperfine structure are determined for each scan individually. For isotopes with multiple scans, a weighted mean for the $A_{S_{1 / 2}}$ factor and the centroid frequency are calculated based on the error of the fits. The uncertainty attributed to the $A_{S_{1 / 2}}$ factor is calculated as the weighted standard deviation of the values. The isotope shifts are determined relative to ${ }^{221} \mathrm{Fr}$, with the uncertainty propagated from the error of the fits, the scatter, and the drift in the centroid frequency of the ${ }^{221} \mathrm{Fr}$ reference scans [41].

\section{A. Spectroscopic studies of ${ }^{204} \mathbf{F r}$}

The hyperfine structure of ${ }^{204} \mathrm{Fr}$ is shown in Fig. 2, measured by detecting the laser ions with the MCP detector as a function of the scanned first-step laser frequency. Five peaks are observed in the spectrum. Considering that only

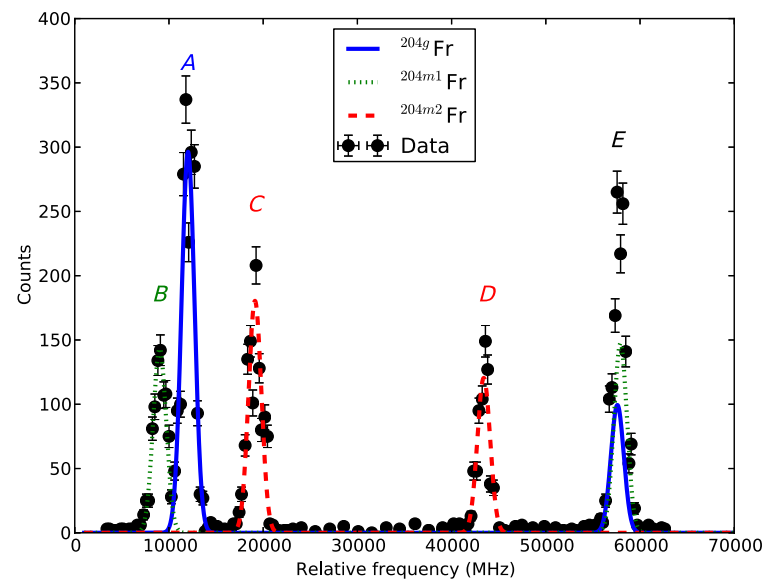

FIG. 2. Collinear resonance ionization spectroscopy of ${ }^{204} \mathrm{Fr}$ relative to ${ }^{221} \mathrm{Fr}$. The hyperfine structure of the $3^{(+)}$ground state of ${ }^{204 g} \mathrm{Fr}$ is shown in blue, the $7^{+}$state of ${ }^{204 m 1} \mathrm{Fr}$ is shown in green, and the $\left(10^{-}\right)$state of ${ }^{204 m 2} \mathrm{Fr}$ is shown in red. 


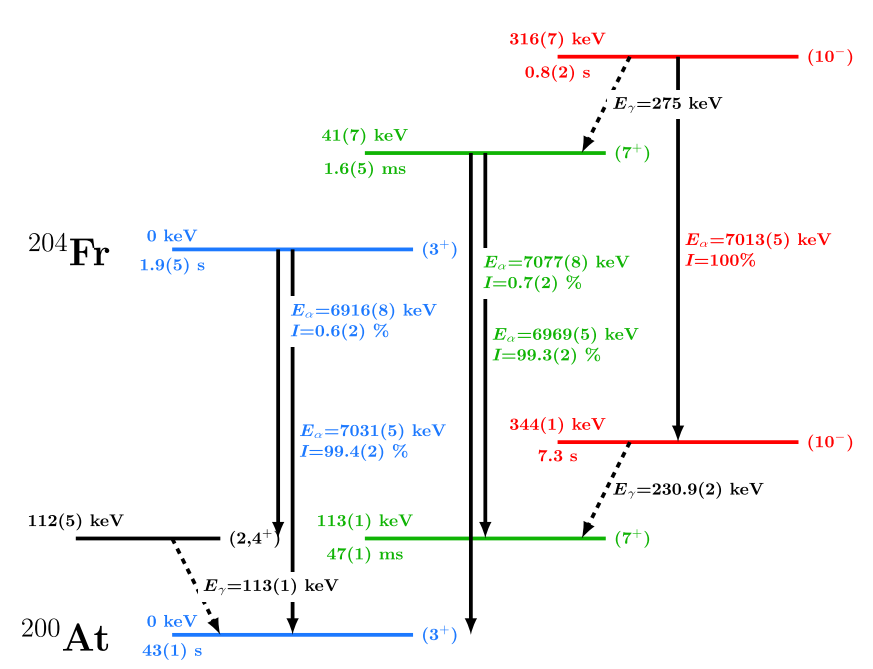

FIG. 3. The radioactive decay of ${ }^{204} \mathrm{Fr}$ and its isomers $[16,18,19]$.

the lower-state splitting is resolved (associated with the $1.5-\mathrm{GHz}$ linewidth of the scanning laser), two hyperfine resonances are expected per nuclear (ground or isomeric) state. Consequently, Fig. 2 contains the hyperfine structure of three long-lived states in ${ }^{204} \mathrm{Fr}$, with one of the resonances unresolved (labeled E). In order to identify the states of the hyperfine resonances, laser-assisted alphadecay spectroscopy is used.

The radioactive decay of the low-lying states in ${ }^{204} \mathrm{Fr}$ is presented in Fig. 3. The characteristic alpha decay of each nuclear state in ${ }^{204} \mathrm{Fr}$ is utilized to identify the hyperfinestructure resonances of Fig. 2. The laser is tuned on resonance with each of the first three hyperfine resonances (labeled $A, B$, and $C$ ), and alpha-decay spectroscopy is performed on each. The alpha-particle energy spectrum of

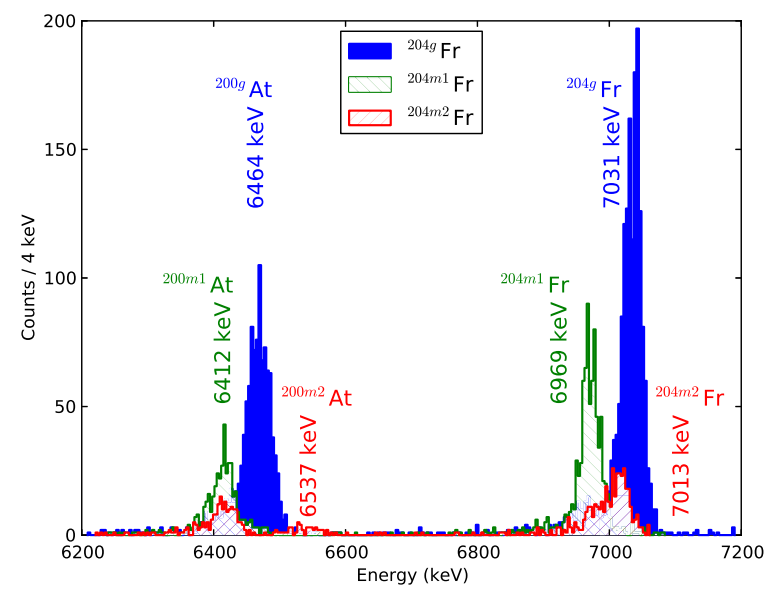

FIG. 4. Alpha-particle spectroscopy of (blue) ${ }^{204 g} \mathrm{Fr}$, (green) ${ }^{204 m 1} \mathrm{Fr}$, and (red) ${ }^{204 m}$ Fr allow the hyperfine peaks in Fig. 2 to be identified. The laser is detuned by $11.503 \mathrm{GHz}$ (peak $A,{ }^{204 g} \mathrm{Fr}$ ), $8.508 \mathrm{GHz}$ (peak $B,{ }^{204 m 1} \mathrm{Fr}$ ), and $18.693 \mathrm{GHz}$ (peak $C,{ }^{204 m 2} \mathrm{Fr}$ ) relative to the centroid frequency of ${ }^{221} \mathrm{Fr}$. these three states is illustrated in Fig. 4. The energy of the alpha particles emitted when the laser is on resonance with an atomic transition of the hyperfine spectrum characteristic of ${ }^{204 g} \mathrm{Fr}$ is shown in blue. This transition occurs at $11.503 \mathrm{GHz}$ (peak $A$ of Fig. 2) relative to the centroid frequency of ${ }^{221}$ Fr. Similarly, the alpha-particle energy spectra for ${ }^{204 \mathrm{~m} 1} \mathrm{Fr}$ and ${ }^{204 \mathrm{~m} 2} \mathrm{Fr}$ are shown in green and red, when the laser is detuned by 8.508 and $18.693 \mathrm{GHz}$ (peaks $B$ and $C$ ) from the reference frequency, respectively. Present in the alpha-particle energy spectrum are the alpha particles emitted from the decay of the ${ }^{204} \mathrm{Fr}$ states $(6950-7050 \mathrm{keV})$ in addition to those emitted from the nuclear states in the daughter isotope ${ }^{200}$ At (6400$6500 \mathrm{keV}$ ). Each state in ${ }^{204} \mathrm{Fr}$ has a characteristic alpha-particle emission energy: $7031 \mathrm{keV}$ for ${ }^{204 g} \mathrm{Fr}$, $6969 \mathrm{keV}$ for ${ }^{204 m 1} \mathrm{Fr}$, and $7013 \mathrm{keV}$ for ${ }^{204 m 2} \mathrm{Fr}$. The identification of the state is confirmed by the presence of the corresponding daughter decays of ${ }^{200 g} \mathrm{At}(6464 \mathrm{keV})$, ${ }^{200 m 1}$ At $(6412 \mathrm{keV})$, and ${ }^{200 m 2}$ At $(6537 \mathrm{keV})$ in the alphaparticle energy spectrum.

An additional alpha-decay measurement is performed on peak $D$ in the hyperfine spectrum of ${ }^{204} \mathrm{Fr}$ (see Fig. 2) at $43.258 \mathrm{GHz}$ relative to the centroid frequency of ${ }^{221} \mathrm{Fr}$. The observation of 7013-keV alpha particles allows this state to be identified as ${ }^{204 m 2} \mathrm{Fr}$. Identification of peak $D$ means the identity of all five hyperfine-structure peaks can be allocated to a state in ${ }^{204} \mathrm{Fr}$ (hence, the hyperfine-structure peak $E$ is the overlapping structure of ${ }^{204 g} \mathrm{Fr}$ and ${ }^{204 m 1} \mathrm{Fr}$ ), allowing analysis of the hyperfine structure of each state.

In addition to the 7031-keV alpha particles of ${ }^{204 m 2} \mathrm{Fr}$, alpha particles of $6969 \mathrm{keV}$ from the decay of ${ }^{204 \mathrm{ml}} \mathrm{Fr}$ are also observed when the laser is on resonance with the ${ }^{204 m 2} \mathrm{Fr}$ state. The decay of the $\left(10^{-}\right)$state to ${ }^{204 m 1} \mathrm{Fr}$ via an E3 internal transition (IT) has been predicted [18] but only recently observed [17]; see Fig. 3. In the study, the conversion electrons from the internal conversion of ${ }^{204 m 2} \mathrm{Fr}$ were tagged with the emitted $6969-\mathrm{keV}$ alpha particles of ${ }^{204 m 1} \mathrm{Fr}$ that followed (with a 5-s correlation time). The coincidences allowed the predicted energy of the 275-keV isomeric transition to be confirmed. During the CRIS experiment, an additional alpha-decay measurement is performed on ${ }^{204 m 2} \mathrm{Fr}$, with the laser detuned by $43.258 \mathrm{GHz}$ relative to the centroid frequency of ${ }^{221} \mathrm{Fr}$; see Fig. 5. This spectrum confirms the presence of the 6969-keV alpha particle (denoted by $*$ ), emitted from the decay of ${ }^{204 m 1} \mathrm{Fr}$. The ultrapure conditions of this measurement allow the first unambiguous extraction of the branching ratios in the decay of ${ }^{204 m 2} \mathrm{Fr}$ : $B_{\alpha}=53(10) \%$ and $B_{\mathrm{IT}}=47(10) \%$ [42].

Decay-assisted laser spectroscopy is also performed on the hyperfine structure of the low-lying states of ${ }^{204} \mathrm{Fr}$. Just as the laser frequency of the resonant 422.7-nm ionization step is scanned and resonant ions are detected in the collinear resonance ionization spectroscopy of ${ }^{204} \mathrm{Fr}$, the same technique is repeated with the measurement of alpha 


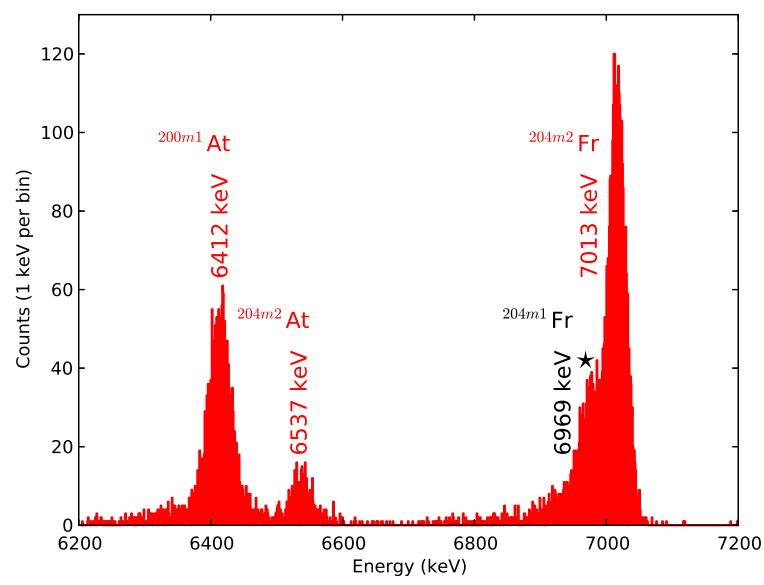

FIG. 5. Alpha-particle spectroscopy of the $\left(10^{-}\right)$state of ${ }^{204 m 2} \mathrm{Fr}$. The decay of ${ }^{204 m 2} \mathrm{Fr}$ to ${ }^{204 m 1} \mathrm{Fr}$ via an E3 IT is observed through the presence of ${ }^{204 \mathrm{~m} 1} \mathrm{Fr}$ alpha particles of $6969 \mathrm{keV}$ (denoted by $*$ ). The laser is detuned by $43.258 \mathrm{GHz}$ relative to the centroid frequency of ${ }^{221} \mathrm{Fr}$.

particles. At each laser frequency, a radioactive-decay measurement of $60 \mathrm{~s}$ is made at the DSS, measuring the alpha particles emitted from the implanted ions. Figure 6 (top) shows the hyperfine peaks associated with each state in ${ }^{204} \mathrm{Fr}$. Measurement of the alpha decay as a function of laser frequency allows production of a matrix of alphaparticle energy versus laser frequency; see Fig. 6.

In order to separate hyperfine structures for each state, an alpha-energy gating is used to maximize the signal-to-noise
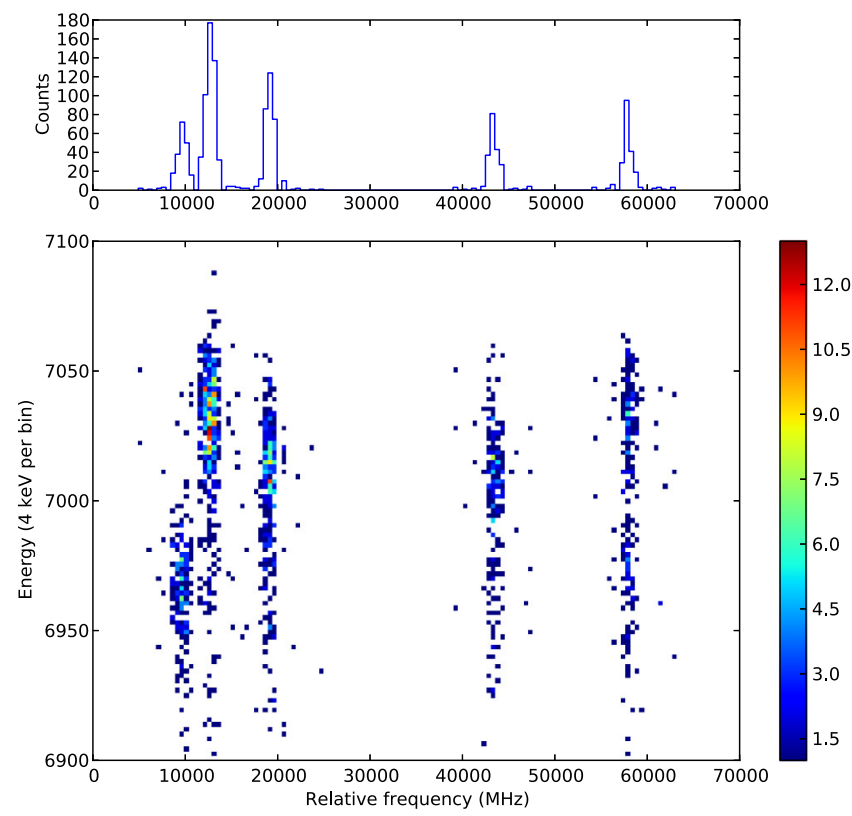

FIG. 6. A two-dimensional histogram of the alpha-particle energy as the hyperfine structure of ${ }^{204} \mathrm{Fr}$ is probed. Top: Projection of the frequency axis. The total number of alpha particles detected at each laser frequency reveals the hyperfine structure of ${ }^{204} \mathrm{Fr}$.

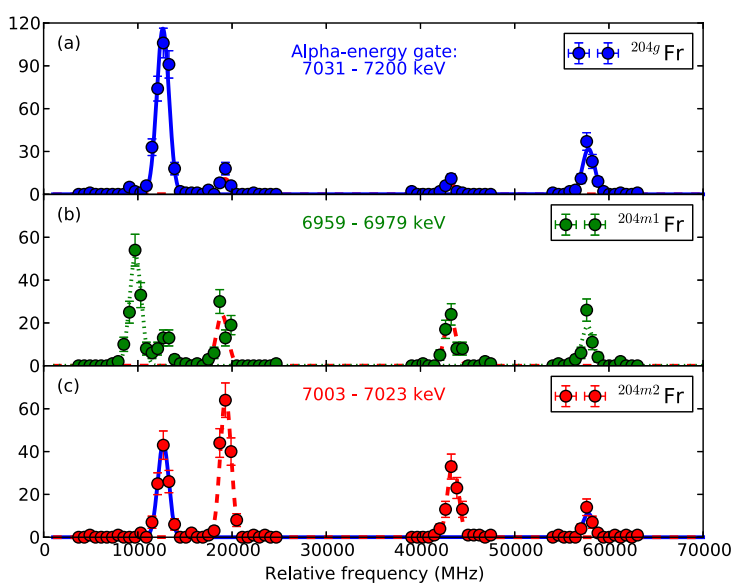

FIG. 7. Alpha-tagged hyperfine structure of (a) the ${ }^{204 g}$ Fr spin $3^{(+)}$ground state, (b) the ${ }^{204 m 1} \mathrm{Fr}$ spin $\left(7^{+}\right)$isomer, and (c) the ${ }^{204 m 2}$ Fr spin $\left(10^{-}\right)$isomer. Additional peaks are discussed in the text.

ratio for the alpha particle of interest. The alpha-energy gates are chosen to be $7031-7200 \mathrm{keV}$ for ${ }^{204 g} \mathrm{Fr}$, 6959$6979 \mathrm{keV}$ for ${ }^{204 m 1} \mathrm{Fr}$, and $7003-7023 \mathrm{keV}$ for ${ }^{204 m 2} \mathrm{Fr}$.

By gating on the characteristic alpha-particle energies of the three states in ${ }^{204} \mathrm{Fr}$, the hyperfine structures of individual isomers become enhanced in the hyperfine spectrum. Figure 7(a) shows the hyperfine structure of ${ }^{204 g}$ Fr, Fig. 7(b) shows ${ }^{204 \mathrm{~m} 1} \mathrm{Fr}$, and Fig. 7(c) shows ${ }^{204 m 2} \mathrm{Fr}$. The presence of ${ }^{204 \mathrm{~m} 2} \mathrm{Fr}$ can be observed in the spectra of ${ }^{204 g}$ Fr due to the overlapping peaks of the alpha energies: The tail of the 7013-keV alpha peak is present in the gate of the ${ }^{204 g} \mathrm{Fr}$ alpha peak. The presence of ${ }^{204 m 2} \mathrm{Fr}$ in the hyperfine-structure spectrum of ${ }^{204 m 1} \mathrm{Fr}$ is attributed to the E3 IT decay of ${ }^{204 m 2} \mathrm{Fr}$ to ${ }^{204 \mathrm{~m} 1} \mathrm{Fr}$ : Alpha particles of energy $6969 \mathrm{keV}$ are observed when on resonance with ${ }^{204 m 2} \mathrm{Fr}$. Additionally, ${ }^{204 g} \mathrm{Fr}$ is present in the ${ }^{204 m 2} \mathrm{Fr}$ spectra due to the similar energies of the 7031- and 7013-keV alpha particles. However, despite the contamination in the hyperfine spectra, each peak is separated sufficiently in frequency to be analyzed independently.

From the resulting hyperfine structures of Fig. 7 produced by the alpha-tagging process (in comparison to the overlapping ion data of Fig. 2), each state of ${ }^{204} \mathrm{Fr}$ can be analyzed individually and the hyperfine factors extracted with better accuracy and reliability. The estimated error of the $A_{S_{1 / 2}}$ factors is $30 \mathrm{MHz}$ on account of the scatter of $A_{S_{1 / 2}}$ values for ${ }^{221} \mathrm{Fr}$. Likewise, an error of $100 \mathrm{MHz}$ is assigned to the isotope shifts.

\section{B. Identification of the hyperfine structure of ${ }^{202} \mathrm{Fr}$}

The hyperfine structure of ${ }^{202} \mathrm{Fr}$ obtained with collinear resonance ionization spectroscopy is presented in Fig. 8. The four hyperfine resonances illustrate the presence of the ground $\left({ }^{202 g} \mathrm{Fr}\right)$ and isomeric $\left({ }^{202 m} \mathrm{Fr}\right)$ states. Identification of these two states is performed with laser-assisted 


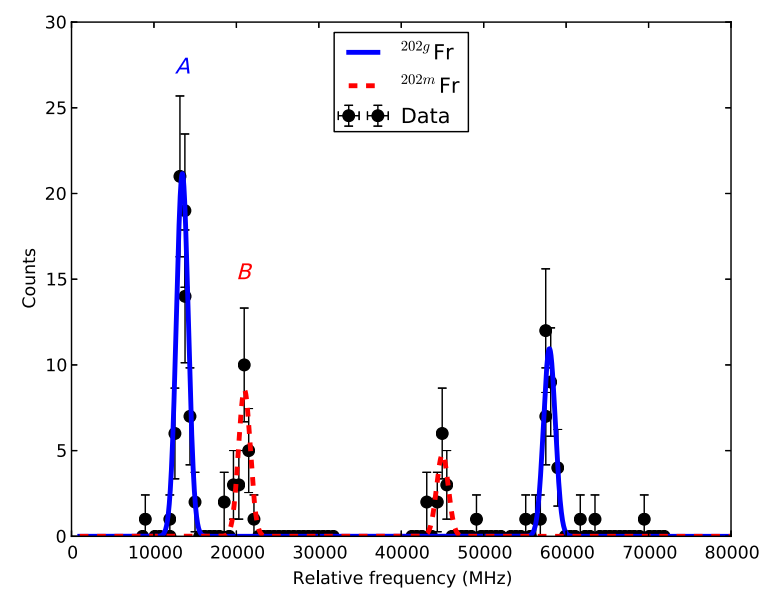

FIG. 8. Collinear resonance ionization spectroscopy of ${ }^{202} \mathrm{Fr}$ relative to ${ }^{221} \mathrm{Fr}$. The hyperfine structure of the $\left(3^{+}\right)$ground state of ${ }^{202 g} \mathrm{Fr}$ is shown as a solid blue line, and the $\left(10^{-}\right)$state of ${ }^{202 m} \mathrm{Fr}$ is shown as a dashed red line.

alpha-decay spectroscopy. According to the literature, the radioactive decay of ${ }^{202 g} \mathrm{Fr}\left[t_{1 / 2}=0.30(5) \mathrm{s}\right]$ emits an alpha particle of energy $7241(8) \mathrm{keV}$, whereas ${ }^{202 m} \mathrm{Fr}$ $\left[t_{1 / 2}=0.29(5) \mathrm{s}\right]$ emits an alpha particle of energy $7235(8) \mathrm{keV}$ [44]. The radioactive decay of the ground and isomeric states of ${ }^{202} \mathrm{Fr}$ is presented in Fig. 9.

The laser is tuned onto resonance with peak $A\left({ }^{202 g} \mathrm{Fr}\right.$, $13.760 \mathrm{GHz}$ relative to the centroid frequency of ${ }^{221} \mathrm{Fr}$ ) and peak $B\left({ }^{202 m} \mathrm{Fr}, 20.950 \mathrm{GHz}\right.$ relative to the centroid frequency of ${ }^{221} \mathrm{Fr}$ ) of Fig. 8 obtained from ion detection. For each position, an alpha-decay measurement is performed, shown in Fig. 10. The alpha particles emitted when the laser is on resonance with an atomic transition characteristic to ${ }^{202 g} \mathrm{Fr}$ are shown in blue, and ${ }^{202 m} \mathrm{Fr}$ in red. Because of the limited statistics of our measurement, and the similarity in energies of the alpha particles (within error), it is impossible to say that alpha particles of different energies are observed in Fig. 10.

Firm identification of the hyperfine components can be achieved, however, by studying the alpha particles emitted

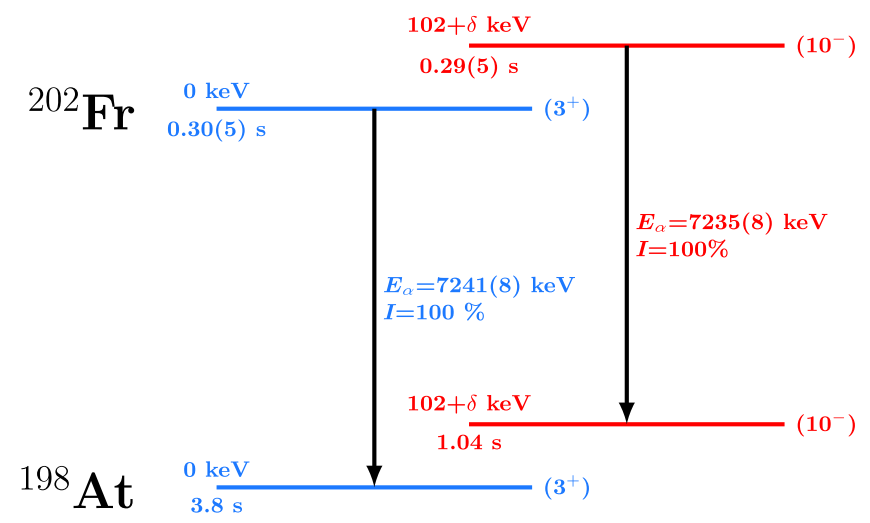

FIG. 9. The radioactive decay of ${ }^{202} \mathrm{Fr}$ and its isomer [43].

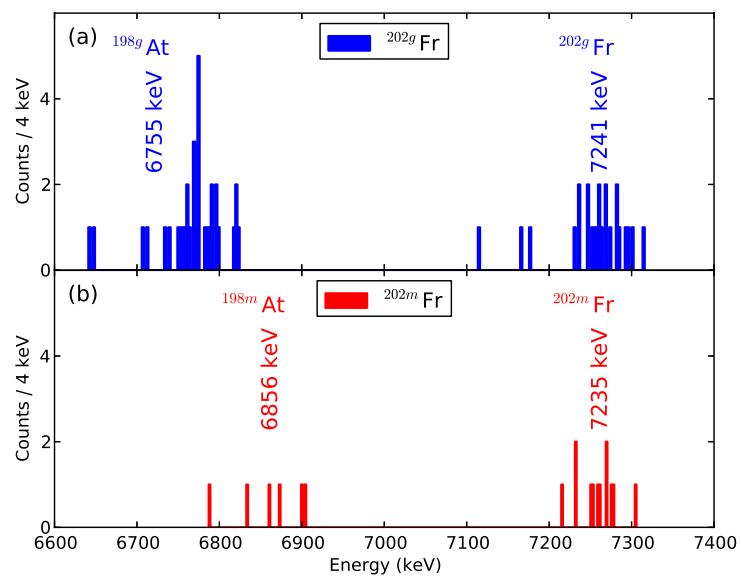

FIG. 10. Alpha-particle spectroscopy of (blue) ${ }^{202 g} \mathrm{Fr}$ and (red) ${ }^{202 m} \mathrm{Fr}$ allow the hyperfine peaks in Fig. 8 to be identified. The laser is detuned by (a) $13.760 \mathrm{GHz}$ (peak $A,{ }^{202 g} \mathrm{Fr}$ ) and (b) $20.950 \mathrm{GHz}$ (peak $B,{ }^{202 m} \mathrm{Fr}$ ) relative to the centroid frequency of ${ }^{221} \mathrm{Fr}$.

by the daughter isotopes ${ }^{198 g, m}$ At. Evident in the spectrum of ${ }^{202 g} \mathrm{Fr}$ are the alpha particles emitted from the decay of the daughter nucleus ${ }^{198 g}$ At with an energy of $6755 \mathrm{keV}$. Similarly, present in the ${ }^{202 m} \mathrm{Fr}$ spectrum are the alpha particles from the decay of ${ }^{198 m}$ At with an energy of $6856 \mathrm{keV}$. The difference in energy of these two alpha peaks illustrates the ability of the CRIS technique to separate the two states and provide pure ground-state and isomeric beams for decay spectroscopy.

\section{Isomer identification of the resonance spectrum of ${ }^{206} \mathrm{Fr}$}

Two sets of data are used in the determination of the nuclear observables from the hyperfine-structure scans. The

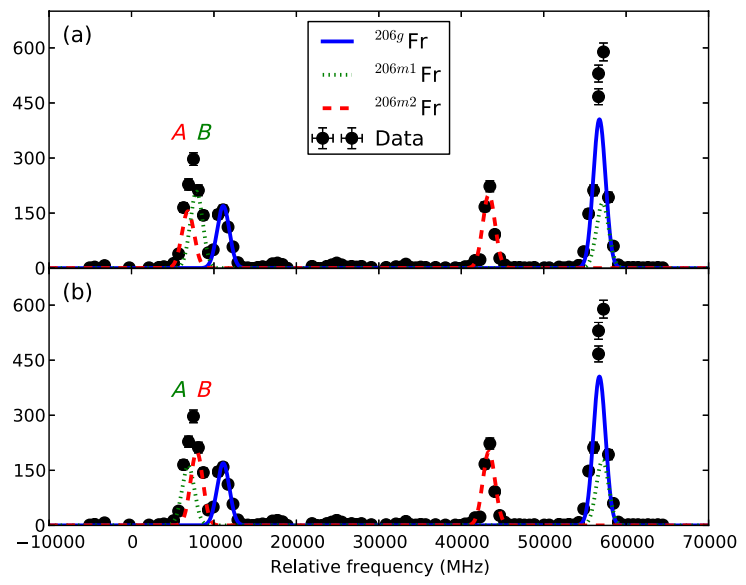

FIG. 11. Collinear resonance ionization spectroscopy of ${ }^{206} \mathrm{Fr}$ relative to ${ }^{221} \mathrm{Fr}$. (a) Option 1: Peak $A$ is assigned to ${ }^{206 m 2} \mathrm{Fr}$ and peak $B$ to ${ }^{206 m 1}$ Fr. (b) Option 2: Peak $A$ is assigned to ${ }^{206 m 1} \mathrm{Fr}$ and peak $B$ to ${ }^{206 m 2} \mathrm{Fr}$. 
TABLE I. Yields of the neutron-deficient francium isotopes at the ISOLDE facility (1.4-GeV protons on a $\mathrm{UC}_{x}$ target). The nuclear-state compositions of the radioactive beams for ${ }^{202,204,206}$ Fr are presented.

\begin{tabular}{lcccr}
\hline \hline & Yield & \multicolumn{3}{c}{ Proportion of beam } \\
$A$ & $($ Ions/s) & Spin 3 $3^{(+)}$ & Spin $\left(7^{(+)}\right)$ & Spin $\left(10^{-}\right)$ \\
\hline 202 & $1 \times 10^{2}$ & $76(14) \%$ & & $24(6) \%$ \\
203 & $1 \times 10^{3}$ & & & \\
204 & $1 \times 10^{4 a}$ & $63(3) \%$ & $27(3) \%$ & $10(1) \%$ \\
205 & $2 \times 10^{5}$ & & & \\
206 & $3 \times 10^{6}$ & $63(7) \%$ & $27(5) \%$ & $9(1) \%$ \\
\hline \hline
\end{tabular}

${ }^{\mathrm{a}}$ Estimate based on yield systematics.

data for the francium isotopes ${ }^{202-206,221} \mathrm{Fr}$ are taken in run I, and the data for ${ }^{202-205,221} \mathrm{Fr}$ are taken in run II. Consistency checks are carried out, allowing ${ }^{206} \mathrm{Fr}$ to be evaluated with respect to the rest of the data set from run II. A detailed description of this analysis can be found in Ref. [42]. In run I, no alpha tagging is available, and consequently, the peaks in the ion-detected hyperfine spectrum need to be identified in a different way. Recent measurements of the ground-state hyperfine structure of ${ }^{206 g} \mathrm{Fr}$ provide the $A_{S_{1 / 2}}$ factor for the splitting of the $7 s^{2} S_{1 / 2}$ state [20]. One peak of the $\left(7^{+}\right)$isomeric state is also identified in this experiment [see Fig. 1(c) of Ref. [20]], allowing the positions of the overlapping resonances to be determined. Therefore only the identities of peaks $A$ and $B$ (shown in Fig. 11) are unknown. Figure 11(a) presents the hyperfine structures when peak $A$ is assigned to ${ }^{206 m 2} \mathrm{Fr}$ and peak $B$ to ${ }^{206 m 1}$ Fr. Figure 11(b) shows the fit when peak $A$ is ${ }^{206 m 1} \mathrm{Fr}$ and peak $B$ is ${ }^{206 m 2} \mathrm{Fr}$. The suggested identity of the two resonances (based on mean-square charge radii and $g$-factor systematics) is discussed in Sec. IV.

\section{Yield measurements}

The yields of the neutron-deficient francium isotopes ${ }^{202-206} \mathrm{Fr}$ are presented in Table I. The quoted yields, scaled ISOLDE-database yields based on an independent yield measurement of ${ }^{202} \mathrm{Fr}$, can be expected to vary by a factor of 2 due to different targets. The quoted value for ${ }^{204} \mathrm{Fr}$ is estimated based on francium-yield systematics. The composition of the beam for ${ }^{202,204,206} \mathrm{Fr}$ is calculated from the ratio of hyperfine-peak intensities (based on the strongest hyperfine-structure resonance) from the CRIS ion data. The composition of the beam for ${ }^{202} \mathrm{Fr}$ is confirmed with the alpha-decay data measured with the DSS.

\section{E. King-plot analysis}

The atomic factors $F$ and $M$ are evaluated by the Kingplot method [45]. The technique combines the previously measured isotope shifts by Coc et al. [7] of the $7 s^{2} S_{1 / 2} \rightarrow$ $7 p^{2} P_{3 / 2}$ transition, with 718-nm laser light, with those made by Duong et al. [10] of the $7 s^{2} S_{1 / 2} \rightarrow 8 p^{2} P_{3 / 2}$

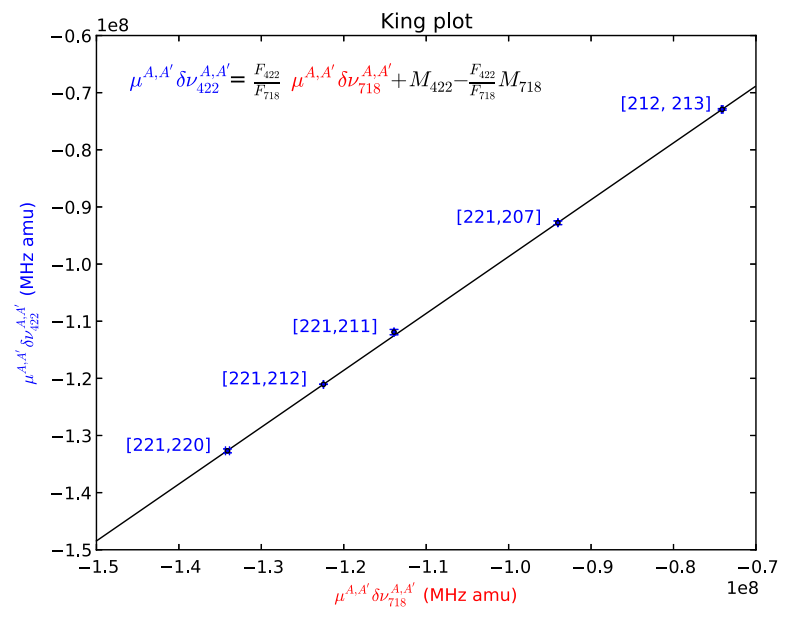

FIG. 12. A King plot for the extraction of atomic factors $F$ and $M$ for the 422.7-nm transition. See the text for details.

transition $(422.7 \mathrm{~nm})$. The isotope shifts of $\delta \nu^{207,221}$ and $\delta \nu^{211,221}$ from this work are combined with $\delta \nu^{220,221}$ and $\delta \nu^{213,212}$ from Ref. [10]. These values are plotted against the corresponding isotope shifts from Coc et al. [7], shown in Fig. 12. The linear fit of the data, and using

$$
\mu^{A, A^{\prime}} \delta \nu_{422}^{A, A^{\prime}}=\frac{F_{422}}{F_{718}} \mu^{A, A^{\prime}} \delta \nu_{718}^{A, A^{\prime}}+M_{422}-\frac{F_{422}}{F_{718}} M_{718},
$$

where $\mu^{A, A^{\prime}}=A A^{\prime} /\left(A^{\prime}-A\right)$, enable the evaluation of $F_{422} / F_{718}=+0.995(3)$ and $M_{422}-\left(F_{422} / F_{718}\right) M_{718}=$ $+837(308) \mathrm{GHzamu}$, respectively. From these values, the atomic factors for the 422.7-nm transition are calculated to be

$$
\begin{aligned}
F_{422} & =-20.67(21) \mathrm{GHz} / \mathrm{fm}^{2}, \\
M_{422} & =+750(330) \mathrm{GHz} \text { amu } .
\end{aligned}
$$

For comparison, the atomic factors evaluated for the 718-nm transition are determined by Dzuba et al. to be $F_{718}=-20.766(208) \mathrm{GHz} / \mathrm{fm}^{2}$ and $M_{718}=-85(113)$ GHz amu [46].

The mass factor is the linear combination of two components: the normal mass shift $K^{\mathrm{NMS}}$ and the specific mass shift $K^{\text {SMS }}$,

$$
M_{422}=K_{422}^{\mathrm{NMS}}+K_{422}^{\mathrm{SMS}},
$$

and is dependent on the frequency of the transition probed. Subtraction of the normal mass shift of the 422.7-nm transition $\left(K_{422}^{\mathrm{NMS}}=+389 \mathrm{GHz} \mathrm{amu}\right)$ from the mass factor $M_{422}$ allows for calculation of the specific mass shift, giving $K_{422}^{\mathrm{SMS}}=+360$ (330) GHzamu. The specific mass shift for the 718-nm line is determined by Dzuba et al. to be $K_{718}^{\text {SMS }}=-314(113) \mathrm{GHz}$ amu [46]. 


\section{F. Hyperfine-structure observables}

Table II presents the hyperfine $A_{S_{1 / 2}}$ factor, isotope shift, change in mean-square charge radius, and magneticmoment values extracted from the CRIS data for the francium isotopes ${ }^{202-206} \mathrm{Fr}$ with reference to ${ }^{221} \mathrm{Fr}$. Additional data for ${ }^{207,211,220} \mathrm{Fr}$ (used in the creation of the King plot of Fig. 12) are included for completeness. All values are deduced using the nuclear spins presented.

The hyperfine $A_{S_{1 / 2}}$ factor is defined as

$$
A=\frac{\mu_{I} B_{e}}{I J}
$$

with $\mu_{I}$ the magnetic dipole moment of the nucleus and $B_{e}$ the magnetic field of the electrons at the nucleus. For each isotope, it is calculated from the weighted mean of $A_{S_{1 / 2}}$ values for isotopes where more than one hyperfinestructure scan is present. A minimum error of $30 \mathrm{MHz}$ is attributed to the $A_{S_{1 / 2}}$-factor values due to the scatter of the measured $A_{S_{1 / 2}}$ for the reference isotope ${ }^{221} \mathrm{Fr}[41,53]$.

The isotope shift $\delta \nu^{A, A^{\prime}}$ between isotopes $A$ and $A^{\prime}$ is expressed as

$$
\delta \nu^{A, A^{\prime}}=M \frac{A^{\prime}-A}{A A^{\prime}}+F \delta\left\langle r^{2}\right\rangle^{A, A^{\prime}} .
$$

As with the $A_{S_{1 / 2}}$ values, the isotope shifts are calculated as the weighted mean of all isotope shifts for a given nucleus. The error on the isotope shift is determined to be $100 \mathrm{MHz}$ due to the long-term drift of the centroid frequency of ${ }^{221} \mathrm{Fr}$ as the experiment progresses and the scan-to-scan scatter in centroid frequency. When the calculated weighted standard deviation of the isotope shift is higher than $100 \mathrm{MHz}$, this error is quoted instead. Combining the extracted $F$ and $M$ atomic factors from the King-plot analysis with the measured isotope shifts, evaluation of the change in mean-square charge radii $\delta\left\langle r^{2}\right\rangle^{A, A^{\prime}}$ between francium isotopes can be performed; see Eq. (6).

The magnetic moment of the isotopes under investigation can be extracted from the known moment of another isotope of the element, using the ratio

$$
\mu=\mu_{\mathrm{ref}} \frac{I A}{I_{\mathrm{ref}} A_{\mathrm{ref}}} .
$$

In this work, calculation of the magnetic moments is evaluated in reference to the magnetic moment of ${ }^{210} \mathrm{Fr}$, measured by Gomez et al. $\left[\mu=+4.38(5) \mu_{N}, I^{\pi}=6^{+}\right.$, $\left.A_{S_{1 / 2}}=+7195.1(4) \mathrm{MHz}[7,52]\right]$. The calculation represents the most accurate measurement of the magnetic moment of a francium isotope to date, due to probing the $9 s^{2} S_{1 / 2}$ hyperfine splitting that has reduced more

TABLE II. Spins, half-lives, $A_{S_{1 / 2}}$ factors, isotope shifts, magnetic moments, and changes in mean-square charge radii of the neutron-

\begin{tabular}{|c|c|c|c|c|c|c|c|c|c|}
\hline \multirow[t]{2}{*}{$A$} & \multirow[t]{2}{*}{$I$} & \multirow{2}{*}{$\begin{array}{r}t_{1 / 2}(\mathrm{~s}) \\
\text { Literature }\end{array}$} & \multicolumn{2}{|c|}{$A_{S_{1 / 2}}(\mathrm{GHz})$} & \multicolumn{2}{|c|}{$\mu\left(\mu_{N}\right)$} & \multirow{2}{*}{$\begin{array}{c}\delta \nu^{A, 221}(\mathrm{GHz}) \\
\text { Experiment }\end{array}$} & \multicolumn{2}{|c|}{$\delta\left\langle r^{2}\right\rangle\left(\mathrm{fm}^{2}\right)$} \\
\hline & & & Experiment & Literature & Experiment & Literature & & Experiment & Literature \\
\hline $202 g$ & $\left(3^{+}\right)$ & $0.30(5)$ & $+12.80(5)$ & & $+3.90(5)$ & & $32.68(10)$ & $-1.596(18)$ & \\
\hline $202 m$ & $\left(10^{-}\right)$ & $0.29(5)$ & $+2.30(3)$ & & $+2.34(4)$ & & $32.57(13)$ & $-1.591(19)$ & \\
\hline 203 & $\left(9 / 2^{-}\right)$ & $0.53(2)$ & $+8.18(3)$ & & $+3.73(4)$ & & $31.32(10)$ & $-1.530(18)$ & \\
\hline $204 g^{\mathrm{a}}$ & $3^{(+)}$ & $1.9(5)$ & $+12.99(3)$ & $+13.1499(43)^{\mathrm{b}}$ & $+3.95(5)$ & $+4.00(5)^{\mathrm{b}, \mathrm{c}}$ & $32.19(10)$ & $-1.571(18)$ & $-1.5542(4)^{b}$ \\
\hline $204 m 1^{\mathrm{a}}$ & $\left(7^{+}\right)$ & $1.6(5)$ & $+6.44(3)$ & & $+4.57(6)$ & & $32.32(10)$ & $-1.577(18)$ & \\
\hline $204 m 2^{\mathrm{a}}$ & $\left(10^{-}\right)$ & $0.8(2)$ & $+2.31(3)$ & & $+2.35(4)$ & & $30.99(10)$ & $-1.513(17)$ & \\
\hline 205 & $9 / 2^{-}$ & $3.96(4)$ & $+8.40(3)$ & $+8.3550(11)^{\mathrm{b}}$ & $+3.83(5)$ & $+3.81(4)^{b, c}$ & $30.21(10)$ & $-1.475(17)$ & $-1.4745(4)^{b}$ \\
\hline $206 g$ & $3^{(+)}$ & $15.9(3)$ & $+13.12(3)$ & $+13.0522(20)^{\mathrm{b}}$ & $+3.99(5)$ & $+3.97(5)^{\mathrm{b}, \mathrm{c}}$ & $30.04(12)$ & $-1.465(17)$ & $-1.4768(4)^{b}$ \\
\hline $206 m 1^{\mathrm{d}}$ & $\left(7^{+}\right)$ & $15.9(3)$ & $+6.61(3)$ & & $+4.69(6)$ & & $30.23(16)$ & $-1.475(18)$ & \\
\hline $206 m 2^{\mathrm{d}}$ & $\left(10^{-}\right)$ & $0.7(1)$ & $+3.50(3)$ & & $+3.55(5)$ & & $23.57(12)$ & $-1.153(14)$ & \\
\hline $206 m 1^{\mathrm{e}}$ & $\left(7^{+}\right)$ & $15.9(3)$ & $+6.74(4)$ & & $+4.79(6)$ & & $29.69(15)$ & $-1.449(17)$ & \\
\hline $206 m 2^{\mathrm{e}}$ & $\left(10^{-}\right)$ & $0.7(1)$ & $+3.40(3)$ & & $+3.45(5)$ & & $24.13(12)$ & $-1.180(14)$ & \\
\hline 207 & $9 / 2^{-}$ & $14.8(1)$ & $+8.48(3)$ & $+8.484(1)^{\mathrm{f}}$ & $+3.87(5)$ & $+3.87(4)^{\mathrm{c}, \mathrm{f}}$ & $28.42(10)$ & $-1.386(16)$ & $-1.386(3)^{g}$ \\
\hline 211 & $9 / 2^{-}$ & 186(1) & $+8.70(6)$ & $+8.7139(8)^{\mathrm{f}}$ & $+3.97(5)$ & $+3.98(5)^{\mathrm{c}, \mathrm{f}}$ & $24.04(10)$ & $-1.171(13)$ & $-1.1779(4)^{g}$ \\
\hline 220 & $1^{+}$ & $27.4(3)$ & $-6.50(4)$ & $-6.5494(9)^{\mathrm{f}}$ & $-0.66(1)$ & $-0.66(1)^{\mathrm{c}, \mathrm{f}}$ & $2.75(10)$ & $-0.134(5)$ & $-0.133(10)^{\mathrm{g}}$ \\
\hline 221 & $5 / 2^{-}$ & $294(12)$ & $+6.20(3)$ & $+6.2046(8)^{\mathrm{f}}$ & $+1.57(2)$ & $+1.57(2)^{\mathrm{c}, \mathrm{f}}$ & 0 & 0 & \\
\hline
\end{tabular}
deficient francium isotopes ${ }^{202-206} \mathrm{Fr}$ with reference to ${ }^{221} \mathrm{Fr}$ for the $7 s^{2} S_{1 / 2} \rightarrow 8 p^{2} P_{3 / 2}$ atomic transition. All $A_{S_{1 / 2}}$-factor and magnetic-moment values are deduced using the nuclear spins presented. The half-life values are taken from Refs. [16,18,47-51].

${ }^{\mathrm{a}}$ Calculated from the alpha-decay gated hyperfine-structure scan of ${ }^{204}$ Fr. See the text for details.

${ }^{\mathrm{b}}$ Literature value taken from Ref. [20]

${ }^{\mathrm{c}}$ Literature magnetic-moment values recalculated in reference to $\mu\left({ }^{210} \mathrm{Fr}\right)$ [52].

${ }^{\mathrm{d}}$ Based on the isomeric identity of the hyperfine resonances of option 1. See the text for details.

${ }^{\mathrm{e}}$ Based on the isomeric identity of the hyperfine resonances of option 2. See the text for details.

${ }^{\mathrm{f}}$ Literature value taken from Ref. [7].

${ }^{\mathrm{g}}$ Literature value taken from Ref. [46]. 
electron-correlation effects than that of the ground state. The current evaluated magnetic moments of the francium isotopes are made in reference to the magnetic moment of ${ }^{211} \mathrm{Fr}$ of Ekström et al. [54].

The hyperfine anomaly for the francium isotopes is generally considered to be of the order of $1 \%$ and is included as a contribution to the error of the hyperfine $A_{S_{1 / 2}}$ factors and magnetic moments [55].

Table II presents the experimental results alongside comparison to the literature of the hyperfine $A_{S_{1 / 2}}$ factor, change in mean-square charge radius, and magneticmoment values. The literature values for ${ }^{204-206} \mathrm{Fr}$ have been taken from Ref. [20] and ${ }^{207,211,220,221} \mathrm{Fr}$ from Ref. [7]. The magnetic-moment values from the literature have been recalculated in reference to $\mu\left({ }^{210} \mathrm{Fr}\right)$ [52], the most accurate measurement to date. The changes in mean-square chargeradii values for ${ }^{207,211,220} \mathrm{Fr}$ have been taken from Ref. [46]. All experimental results are in broad agreement with those of the literature.

\section{DISCUSSION}

\section{A. Charge radii of the neutron-deficient francium}

Located between radon and radium, francium $(Z=87)$ has five valence protons occupying the $\pi 1 h_{9 / 2}$ orbital, according to the shell model of spherical nuclei. Below the $N=126$ shell closure, the neutron-deficient francium isotopes are studied down to ${ }^{202} \mathrm{Fr}(N=115)$. The changes in mean-square charge radii for the francium and lead isotopes are presented in Fig. 13. The data of francium show the charge radii of ${ }^{207-213} \mathrm{Fr}$ reevaluated by Dzuba et al. [46] alongside the CRIS values, which extends the data set to ${ }^{202} \mathrm{Fr}$. The blue data points show the francium

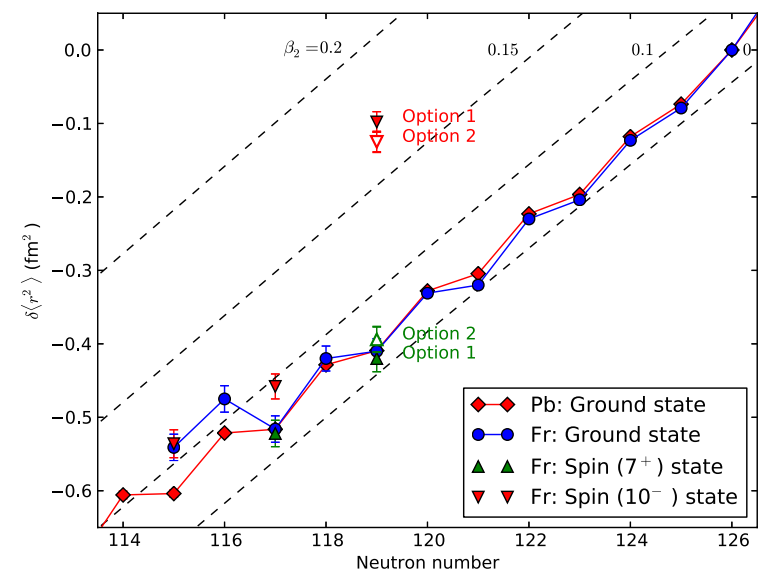

FIG. 13. Mean-square charge radii of the francium (circle) isotopes [54] presented alongside the lead (diamond) isotopes [56]. The dashed lines represent the prediction of the droplet model for given isodeformation [57]. The data are calibrated by using $\beta_{2}\left({ }^{213} \mathrm{Fr}\right)=0.062$, evaluated from the energy of the $2_{1}^{+}$ state in ${ }^{212} \operatorname{Rn}$ [58]. Options 1 and 2 for the $\left(7^{+}\right)$and $\left(10^{-}\right)$states in ${ }^{206} \mathrm{Fr}$ are based on the isomeric identification given in Fig. 11. ground states, while the $\left(7^{+}\right)$isomeric states are in green and the $\left(10^{-}\right)$states in red. The error bars attributed to the CRIS values are propagated from the experimental error of the isotope shift and the systematic error associated with the atomic factors $F_{422}$ and $M_{422}$. The systematic error is the most significant contribution to the uncertainty associated with the mean-square charge radii and not that arising from the isotope shift. The francium data are presented with the lead data of Anselment et al. [56] to illustrate the departure from the spherical nucleus. The changes in mean-square charge radii of the francium isotopes have been overlapped with the charge radii of the lead isotopes, by using ${ }^{213} \mathrm{Fr}$ $(N=126)$ and ${ }^{208} \mathrm{~Pb}(N=126)$ as reference points. The dashed isodeformation lines represent the prediction of the droplet model for the francium isotopes [57]. The data are calibrated using $\beta_{2}\left({ }^{213} \mathrm{Fr}\right)=0.062$, evaluated from the energy of the $2_{1}^{+}$state in ${ }^{212} \mathrm{Rn}$ [58].

The doubly magic ${ }^{208} \mathrm{~Pb}$ represents a model spherical nucleus, with the shape of the nucleus remaining spherical with the removal of neutrons from the closed $N=126$ shell. This trend is observed until $N=114$, where a small deviation from the spherical droplet model (isodeformation line $\beta_{2}=0.0$ ) is interpreted as enhanced collectivity due to the influence of particle-hole excitations across the $Z=82$ shell closure [59]. The change in mean-square charge radii for the francium isotopes shows agreement with the lead data as the $\nu 3 p_{3 / 2}, \nu 2 f_{5 / 2}$, and $\nu 3 p_{1 / 2}$ orbitals are progressively depleted. The deviation from sphericity at $N=116$ with ${ }^{203} \mathrm{Fr}$ marks the onset of collective behavior. The spectroscopic quadrupole moments are not measured in this work, since they require a laser linewidth of $<100 \mathrm{MHz}$. Measurement of the quadrupole moment will provide information on the static deformation component of the change in mean-square charge radii, allowing better understanding of this transition region.

Recent laser-spectroscopy measurements on the groundstate properties of ${ }^{204,205,206} \mathrm{Fr}$ suggest this deviation occurs earlier, at ${ }^{206} \mathrm{Fr}(N=119)$ [20]. In Ref. [20], a more pronounced odd-even staggering is observed in relation to the lead isotopes, where the mean-square charge radius of ${ }^{205} \mathrm{Fr}$ is larger than that of ${ }^{206} \mathrm{Fr}$. The CRIS experiment observes a smaller mean-square charge radius of ${ }^{205} \mathrm{Fr}$ in comparison to ${ }^{206} \mathrm{Fr}$, the deviation from the lead isotopes occurring at ${ }^{203} \mathrm{Fr}$ instead. However, both experiments are in broad agreement within errors down to $N=117$.

Figure 13 presents the two options of the mean-square charge radii of ${ }^{206 m 1} \mathrm{Fr}$ and ${ }^{206 m 2} \mathrm{Fr}$ (as defined by their hyperfine-peak identities in Fig. 11). Option 1 is favored over option 2 due to the smaller mean-square charge radii of ${ }^{206 m 1} \mathrm{Fr}$ (compared to ${ }^{206 g} \mathrm{Fr}$ ) agreeing with the systematics of the states in ${ }^{204} \mathrm{Fr}$. As seen in Fig. 13, ${ }^{206 g} \mathrm{Fr}$ $(N=119)$ overlaps with the lead data within errors. The large change in the mean-square charge radius of ${ }^{206 m 2} \mathrm{Fr}$ suggests a highly deformed state for the $\left(10^{-}\right)$isomer. 


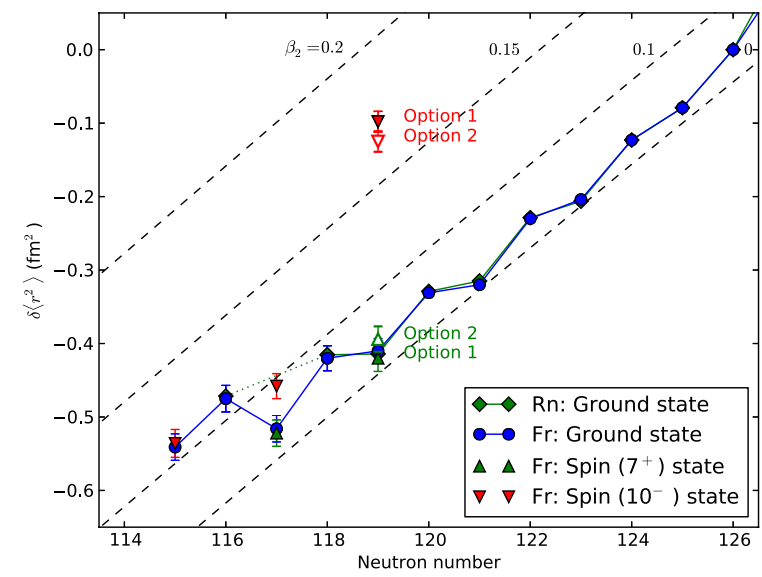

FIG. 14. Mean-square charge radii of the francium (circle) isotopes [54] presented alongside the radon (diamond) isotopes [60]. The dashed lines represent the prediction of the droplet model for given isodeformation [57]. The data are calibrated using $\beta_{2}\left({ }^{213} \mathrm{Fr}\right)=0.062$, evaluated from the energy of the $2_{1}^{+}$ state in ${ }^{212} \operatorname{Rn}$ [58]. Options 1 and 2 for the $\left(7^{+}\right)$and $\left(10^{-}\right)$states in ${ }^{206} \mathrm{Fr}$ are based on the isomeric identification given in Fig. 11.

The mean-square charge radii of francium are overlaid with the radon $(Z=86)$ charge radii of Borchers et al. (down to $N=116$, with the exception of $N=117$ ) [60] in Fig. 14. The mean-square charge radii of radon have been calibrated to the francium pair $\delta\left\langle r^{2}\right\rangle^{211,213}$ to account for the uncertainty in $F$ and $M$ for the optical transition probed. (The original isotope shifts are presented graphically.)

TABLE III. Extracted $\beta_{2}$ values. Experiment: The droplet model [57] is used to extract the rms values for $\beta_{2}$ from the change in mean-square charge radii. The charge-radii values are calibrated using $\beta_{2}\left({ }^{213} \mathrm{Fr}\right)=0.062$, as before. Literature: $\beta_{2}$ values are extracted from the quadrupole moments of Ref. [20]. See the text for details.

\begin{tabular}{lccc}
\hline \hline$A$ & $\mathrm{c}$ & $\begin{array}{c}\left\langle\beta_{2}^{2}\right\rangle^{1 / 2} \\
\text { Experiment }\end{array}$ & $\begin{array}{c}\beta_{2} \\
\text { Literature }\end{array}$ \\
\hline $202 g$ & $\left(3^{+}\right)$ & 0.11 & \\
$202 m$ & $\left(10^{-}\right)$ & 0.11 & \\
203 & $\left(9 / 2^{-}\right)$ & 0.11 & $-0.0140(14)$ \\
$204 g$ & $3^{(+)}$ & $0.06^{\mathrm{a}}$ & \\
$204 m 1$ & $\left(7^{+}\right)$ & $0.06^{\mathrm{a}}$ & $-0.0204(2)$ \\
$204 m 2$ & $\left(10^{-}\right)$ & $0.09^{\mathrm{a}}$ & $-0.0269(8)$ \\
205 & $9 / 2^{-}$ & 0.08 & \\
$206 g$ & $3^{(+)}$ & 0.05 & \\
$206 m 1$ & $\left(7^{+}\right)$ & $0.04^{\mathrm{b}}$ & \\
$206 m 2$ & $\left(10^{-}\right)$ & $0.17^{\mathrm{b}}$ & \\
$202 m 1$ & $\left(7^{+}\right)$ & $0.07^{\mathrm{c}}$ & \\
$202 m 2$ & $\left(10^{-}\right)$ & $0.17^{\mathrm{c}}$ & \\
\hline \hline
\end{tabular}

${ }^{\mathrm{a}}$ Calculated from the alpha-decay gated hyperfine-structure scan of ${ }^{204}$ Fr. See the text for details.

${ }^{b}$ Based on the isomeric identity of the hyperfine resonances of option 1. See the text for details.

${ }^{\mathrm{c}}$ Based on the isomeric identity of the hyperfine resonances of option 2. See the text for details.
Despite the uncertainty, the agreement between the mean-square charge radii of the francium and radon data is clear. The addition of a single $\pi 1 h_{9 / 2}$ proton outside the radon even- $Z$ core does not affect the charge-radii trend, suggesting the valence proton acts as a spectator particle.

Table III presents a comparison of $\beta_{2}$ values with the literature. The droplet model [57] is used to extract the rms values for $\beta_{2}$ (column 3 ) from the change in mean-square charge radii [calibrated using $\beta_{2}\left({ }^{213} \mathrm{Fr}\right)=0.062$, as before]. Column 4 presents $\beta_{2}$ values extracted from the quadrupole moments of Ref. [20]. The larger $\beta_{2}$ values extracted from the mean-square charge radii, compared to those extracted from the quadrupole moments, suggest that the enhanced collectivity observed in Figs. 13 and 14 is due to a large dynamic component of the nuclear deformation.

\section{B. Interpretation of the nuclear $\boldsymbol{g}$ factors}

Figures 15 and 17 show the experimental $g$ factors for odd- $A$ and even- $A$ francium isotopes, respectively. These plots present the CRIS data alongside the data from Ekström et al. [54]. The Ekström et al. data have been reevaluated with respect to the $\mu\left({ }^{210} \mathrm{Fr}\right)$ measurement of Gomez et al. [52].

In Fig. 15, the blue line represents the empirical $g$ factor $\left(g_{\text {emp }}\right)$ of the odd- $A$ isotopes for the single-particle occupation of the valence proton in the $\pi 1 h_{9 / 2}$ orbital. $g_{\text {emp }}\left(\pi 1 h_{9 / 2}\right)$ is determined from the magnetic moment of the single-particle state in ${ }^{209} \mathrm{Bi}$ [61]. Similarly, $g_{\text {emp }}\left(\pi 3 s_{1 / 2}\right)$ is estimated from the magnetic moment of the single-hole ground state in ${ }^{207} \mathrm{Tl}$ [62]. From $N=126$ to 116 , every isotope has a $g$ factor consistent with the proton occupying the $\pi 1 h_{9 / 2}$ orbital. The $g$ factor indicates that the $9 / 2^{-}$state remains the ground state, and the $\left(\pi 3 s_{1 / 2}^{-1}\right)_{1 / 2^{+}}$ proton-intruder state has not yet inverted. This lowering in energy of the $\pi 3 s_{1 / 2}$ state to become the ground state would

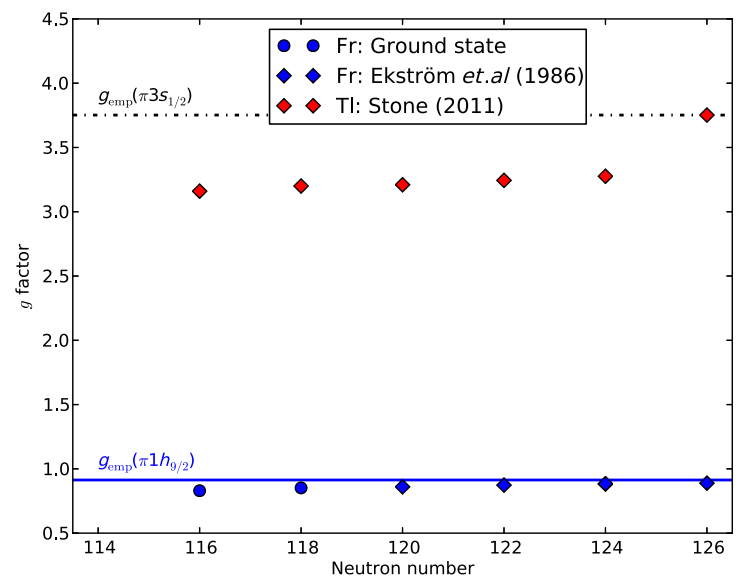

FIG. 15. $g$ factors for francium (solid blue line and symbols) $[52,54]$ and thallium (dot-dashed red line and symbols) isotopes with odd $A$ [63]. The $g$ factors for the $\pi 3 s_{1 / 2}$ and $\pi 1 h_{9 / 2}$ proton orbitals have been calculated empirically. See the text for details. 


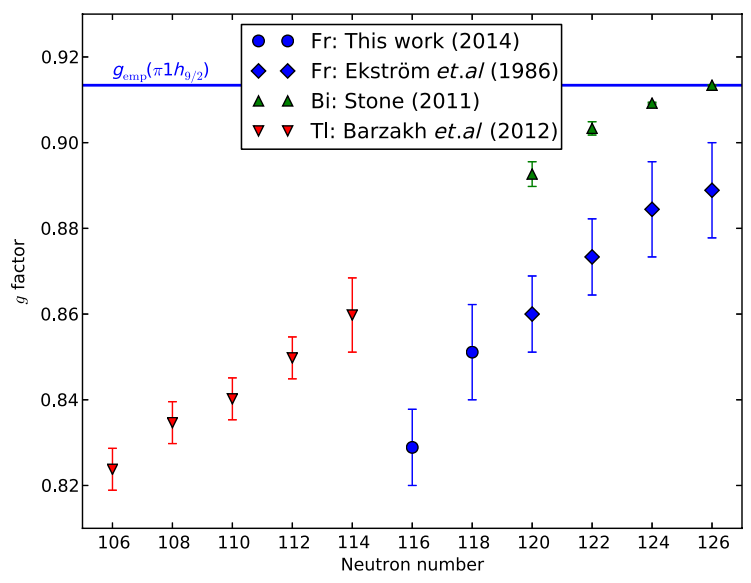

FIG. 16. Close-up of the $g$ factors for francium (blue line and symbols) [52,54], bismuth (green symbols) [63], and thallium (red symbols) isotopes [65] with odd $A$. The $g$ factor for the $\pi 1 h_{9 / 2}$ proton orbital has been calculated empirically. See the text for details.

be apparent in the sudden increase in $g$ factor of the ground state, as illustrated by the black $g_{\mathrm{emp}}\left(\pi 3 s_{1 / 2}\right)$ line.

Figure 15 highlights the robustness of the $Z=82$ and $N=126$ shell closure with a shell-model description valid over a range of isotopes. A close-up of $g_{\mathrm{emp}}\left(1 \pi 1 h_{9 / 2}\right)$ in Fig. 16 illustrates that the $g$ factor is sensitive to bulk nuclear effects. The departure from the $g_{\mathrm{emp}}\left(1 \pi 1 h_{9 / 2}\right)$ line shows the sensitivity of the $g$ factor to second-order core polarization in the odd- $A$ thallium, bismuth, and francium isotopes. The systematic decrease in $g$ factor of francium is attributed to second-order core polarization associated with the presence of five valence particles, compared to one particle (hole) in the bismuth (thallium) isotopes, enough to significantly weaken the shell closure. The linear trend

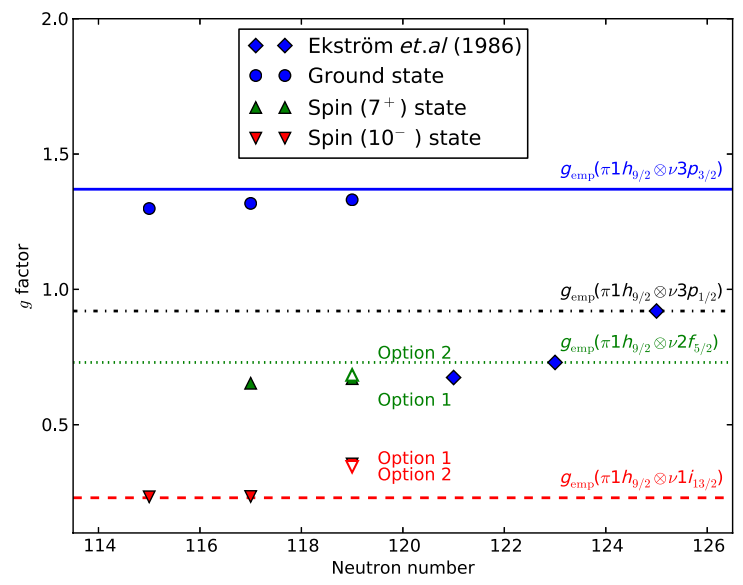

FIG. 17. $g$ factors for francium isotopes with even $A$ [54]: ground state (solid blue line and symbols), spin $\left(7^{+}\right)$state (dotted green line and symbols), and spin $\left(10^{-}\right)$state (dashed red line and symbols). The $g$ factors for the coupling of the proton and neutron orbitals have been calculated empirically. See the text for details. observed in bismuth, thallium, and francium (until $N=118$ ) is suggested to be related to the opening of the neutron shell, yet allowing for more neutron and protonneutron correlations.

Further measurements toward the limit of stability are needed to better understand the prediction of the inversion of the $\pi 3 s_{1 / 2}$ intruder orbital with the $\pi 1 h_{9 / 2}$ ground state. A remeasurement of ${ }^{203} \mathrm{Fr}$ could determine the presence of the spin $1 / 2^{+}$isomer $\left[t_{1 / 2}=43(4) \mathrm{ms}\right.$ [19]], which is not observed during this experiment.

The $g$ factors for the odd-odd francium isotopes are presented in Fig. 17. With the coupling of the single valence proton in the $\pi 1 h_{9 / 2}$ orbital with a valence neutron, a large shell-model space is available. The empirically calculated $g$ factors for the coupling of the $\pi 1 h_{9 / 2}$ proton with the valence neutrons are denoted by the colored lines. These $g$ factors are calculated from the additivity relation

$$
g=\frac{1}{2}\left[g_{p}+g_{n}+\left(g_{p}-g_{n}\right) \frac{j_{p}\left(j_{p}+1\right)-j_{n}\left(j_{n}+1\right)}{I(I+1)}\right],
$$

as outlined by Neyens [64]. The empirical $g$ factors of the odd valence neutrons are calculated from the magnetic moments of neighboring nuclei: ${ }^{201} \mathrm{Po}$ for the blue $g_{\mathrm{emp}}\left(\pi 1 h_{9 / 2} \otimes \nu 3 p_{3 / 2}\right)$ and red $g_{\mathrm{emp}}\left(\pi 1 h_{9 / 2} \otimes \nu 1 i_{13 / 2}\right)$ lines [66], ${ }^{213} \mathrm{Ra}$ for the black $g_{\mathrm{emp}}\left(\pi 1 h_{9 / 2} \otimes \nu 3 p_{1 / 2}\right)$ line, and ${ }^{211} \mathrm{Ra}$ for the green $g_{\mathrm{emp}}\left(\pi 1 h_{9 / 2} \otimes \nu 2 f_{5 / 2}\right)$ line [67]. The empirical $g$ factors for the valence proton in the $\pi 1 h_{9 / 2}$ orbital are calculated from the magnetic moment of the closest odd- $A$ francium isotope $\left({ }^{203} \mathrm{Fr}\right.$ and ${ }^{213} \mathrm{Fr}$, respectively) from the CRIS data.

The ground states of ${ }^{202,204,206} \mathrm{Fr}$ display similar $g$ factors, with the valence proton and neutron coupling to give a spin $3^{(+)}$state. The tentative configuration in the literature of $\left(\pi 1 h_{9 / 2} \otimes \nu 2 f_{5 / 2}\right)_{3^{+}}$for ${ }^{202 g} \mathrm{Fr}$ is based on the configuration of the $\left(3^{+}\right)$state in ${ }^{194} \mathrm{Bi}$ (from favored Fr-AtBi alpha-decay chain systematics) [44]. Similarly, the assignment of the same configuration for ${ }^{204 g} \mathrm{Fr}$ and ${ }^{206 g} \mathrm{Fr}$ is based on the alpha-decay systematics of neighboring nuclei ${ }^{196,198} \mathrm{Bi}$. However, the initial assignment of ${ }^{194 g} \mathrm{Bi}$ is declared to be either $\left(\pi 1 h_{9 / 2} \otimes \nu 2 f_{5 / 2}\right)_{3^{+}}$or $\left(\pi 1 h_{9 / 2} \otimes \nu 3 p_{3 / 2}\right)_{3^{+}}$[68]. From the $g$ factors of the ground states of ${ }^{202,204,206} \mathrm{Fr}$, it is clear that the configuration of these states is indeed $\left(\pi 1 h_{9 / 2} \otimes \nu 3 p_{3 / 2}\right)_{3^{+}}$.

Figure 17 also presents the $g$ factors of ${ }^{206 m 1} \mathrm{Fr}$ and ${ }^{206 m 2} \mathrm{Fr}$ for options 1 and 2 (see Fig. 11). The first isomeric states of ${ }^{204,206} \mathrm{Fr}\left(7^{+}\right)$have a valence neutron that occupies the $\nu 2 f_{5 / 2}$ state. This coupling of the proton and particle with the neutron and hole results in a $\left(\pi 1 h_{9 / 2} \otimes \nu 2 f_{5 / 2}\right)_{7^{+}}$ configuration [69]. For ${ }^{202 m} \mathrm{Fr},{ }^{204 m 2} \mathrm{Fr}$, and ${ }^{206 m 2} \mathrm{Fr}$, the particle-proton-neutron-hole coupling results in a tentative $\left(\pi 1 h_{9 / 2} \otimes \nu 1 i_{13 / 2}\right)_{10^{-}}$configuration assignment for each isomer [70]. However, while the agreement of the $g$ factors 
of the spin $\left(10^{-}\right)$state in ${ }^{202,204} \mathrm{Fr}$ points to a $\nu 1 i_{13 / 2}$ occupancy, the observed value for ${ }^{206 m 2} \mathrm{Fr}$ is in disagreement with the $g$ factor of such a $\left(10^{-}\right)$state. The charge radius of ${ }^{206 \mathrm{~m} 2} \mathrm{Fr}$ indicates a highly deformed configuration, where the single-particle description of the nucleus is no longer valid. The value of the charge radius is consistent with the $g$ factor of this state: It is no longer obeying a simple shell-model description. The observation leads to the conclusion that while a $\left(\pi 1 h_{9 / 2} \otimes \nu 1 i_{13 / 2}\right)_{10^{-}}$configuration for ${ }^{206 m 2} \mathrm{Fr}$ is suggested, the charge radii and magnetic moment point to a drastic change in the structure of this isomeric state.

For completeness, the configurations of the odd-odd francium isotopes ${ }^{208,210,212} \mathrm{Fr}$ are presented. The coupling of the valence proton and neutron in the $\pi 1 h_{9 / 2}$ and $\nu 2 f_{5 / 2}$ orbitals in ${ }^{208} \mathrm{Fr}$ and ${ }^{210} \mathrm{Fr}$ leads to $\left(\pi 1 h_{9 / 2} \otimes \nu 2 f_{5 / 2}\right)_{7+}$ and $\left(\pi 1 h_{9 / 2} \otimes \nu 2 f_{5 / 2}\right)_{6+}$ configurations, respectively [71]. With the $\nu 2 f_{5 / 2}$ neutron orbital fully occupied, the valence neutron in ${ }^{212} \mathrm{Fr}$ occupies the $\nu 3 p_{1 / 2}$ orbital, resulting in a $\left(\pi 1 h_{9 / 2} \otimes \nu 3 p_{1 / 2}\right)_{5+}$ configuration [72].

The agreement of the experimental and empirical $g$ factors, as shown in Figs. 15-17, illustrates the suitability of the single-particle description of the neutron-deficient francium isotopes, with the exception of the $\left(10^{-}\right)$state in ${ }^{206 m 2} \mathrm{Fr}$. A model-independent spin and spectroscopic quadrupole-moment determination is needed to clarify the nature of this isomeric state. The neutron-deficient francium isotopes display a single-particle nature where the additivity relation is still reliable.

\section{CONCLUSION AND OUTLOOK}

The hyperfine structures and isotope shifts of the neutron-deficient francium isotopes ${ }^{202-206} \mathrm{Fr}$ with reference to ${ }^{221} \mathrm{Fr}$ were measured with collinear resonance ionization spectroscopy, with the change in mean-square charge radii and magnetic moments extracted. The selectivity of the alpha-decay patterns allowed the unambiguous identification of the hyperfine components of the low-lying isomers of ${ }^{202,204} \mathrm{Fr}$ for the first time.

The resonant atomic transition of $7 s^{2} S_{1 / 2} \rightarrow 8 p^{2} P_{3 / 2}$ was probed and the hyperfine $A_{S_{1 / 2}}$ factor measured. A King-plot analysis of the 422.7-nm transition in francium allowed the atomic factors to be calibrated. The field and mass factors were determined to be $F_{422}=-20.670(210)$ $\mathrm{GHz} / \mathrm{fm}^{2}$ and $M_{422}=+750$ (330) GHz amu, respectively.

The novel technique of decay-assisted laser spectroscopy in a collinear geometry was performed on the isotopes ${ }^{202,204} \mathrm{Fr}$. The decay-spectroscopy station was utilized to identify the peaks in the hyperfine spectra of ${ }^{202,204} \mathrm{Fr}$. Alpha tagging the hyperfine-structure scan of ${ }^{204} \mathrm{Fr}$ allowed the accurate determination of the nuclear observables of the three low-lying isomeric states and the determination of the branching ratios in the decay of ${ }^{204 m 2} \mathrm{Fr}$.

Analysis of the change in mean-square charge radii suggests an onset of collectivity that occurs at ${ }^{203} \mathrm{Fr}$
( $N=116)$. However, measurement of the spectroscopic quadrupole moment is required to determine the nature of the deformation (static or dynamic). The magnetic moments suggest that the single-particle description of the neutrondeficient francium isotopes still holds, with the exception of the $\left(10^{-}\right)$isomeric state of ${ }^{206 \mathrm{~m} 2} \mathrm{Fr}$. Based on the systematics of the region, the tentative assignments of the hyperfinestructure peaks in ${ }^{206} \mathrm{Fr}$ result in magnetic moments and mean-square charge radii that suggest a highly deformed state. Laser-assisted nuclear-decay spectroscopy of ${ }^{206} \mathrm{Fr}$ would unambiguously determine their identity.

The occupation of the valence proton in the $\pi 1 h_{9 / 2}$ orbital has been suggested for all measured isotopes down to ${ }^{202} \mathrm{Fr}$, indicating the $\left(\pi 1 s_{1 / 2}^{-1}\right)_{1 / 2^{+}}$intruder state does not yet invert with the $\pi 1 h_{9 / 2}$ orbital as the ground state. Further measurements of the very neutron-deficient francium isotopes toward ${ }^{199} \mathrm{Fr}$ are required to fully determine the nature of the proton-intruder state. A laser linewidth of $1.5 \mathrm{GHz}$ was enough to resolve the lower-state $\left(7 s^{2} S_{1 / 2}\right)$ splitting of the hyperfine structure and measure the $A_{S_{1 / 2}}$ factor. In the future, the inclusion of a narrow-band laser system for the resonantexcitation step will enable the resolution of the upper-state $\left(8 p^{2} P_{3 / 2}\right)$ splitting, providing the hyperfine $B_{P_{3 / 2}}$ factor. Measurement of the upper-state splitting will allow extraction of the spectroscopic quadrupole moment and determination of the nature of the deformation.

Successful measurement of ${ }^{202} \mathrm{Fr}$ was performed during this experiment, with a yield of 100 atoms/s. By pushing the limits of laser spectroscopy, further measurements of ${ }^{201} \mathrm{Fr}$ (with a yield of 1 atom/s) and ${ }^{200} \mathrm{Fr}$ (less than 1 atom/s) are thought to be possible. The ground state $\left(9 / 2^{-}\right)$ of ${ }^{201} \mathrm{Fr}$ has a half-life of $53 \mathrm{~ms}$ and its isomer $\left(1 / 2^{+}\right)$a half-life of $19 \mathrm{~ms}$. By increasing the sensitivity of the CRIS technique, the presence of the $1 / 2^{+}$isomers in ${ }^{201,203} \mathrm{Fr}$ can be confirmed. A positive identification will lead to nuclearstructure measurements that will determine (along with the verification of nuclear spin) the magnetic moments that are sensitive to the single-particle structure and thus to the $\left(\pi 3 s_{1 / 2}\right)_{1 / 2^{+}}$proton-intruder nature of these states. With sufficient resolution $(<100 \mathrm{MHz})$, the spectroscopic quadrupole moment of these neutron-deficient states (with $I \geq 1$ ) will be directly measurable and the time-averaged static deformation can be determined.

The successful measurements performed by the CRIS experiment demonstrate the high sensitivity of the collinear resonance ionization technique. The decay-spectroscopy station provides the ability to identify overlapping hyperfine structures and eventually perform laser-assisted nucleardecay-spectroscopy measurements on pure ground- and isomeric-state beams [32,73].

\section{ACKNOWLEDGMENTS}

The authors extend their thanks to the ISOLDE team for providing the beam, the GSI target lab for producing the carbon foils, and IKS-KU Leuven and The University of 
Manchester machine shops for their work. This work was supported by the IAP Project P7/23 of the OSTC Belgium (BRIX network) and by the FWO-Vlaanderen (Belgium). The Manchester group was supported by the STFC Consolidated Grant No. ST/F012071/1 and Continuation Grant No. ST/J000159/1. K. T. F. was supported by STFC Advanced Fellowship Scheme Grant No. ST/G006415/1. The authors would also like to thank Ed Schneiderman for continued support through donations to the Physics Department at NYU.

[1] B. Cheal and K. T. Flanagan, Progress in Laser Spectroscopy at Radioactive Ion Beam Facilities, J. Phys. G 37, 113101 (2010).

[2] K. Blaum, J. Dilling, and W. Nörtershäuser, Precision Atomic Physics Techniques for Nuclear Physics with Radioactive Beams, Phys. Scr. T152, 014017 (2013).

[3] T. J. Procter and K. T. Flanagan, First On-line Results from the CRIS (Collinear Resonant Ionisation Spectroscopy) Beam Line at ISOLDE, Hyperfine Interact. 216, 89 (2013).

[4] S. Liberman et al., Laser Optical Spectroscopy on Francium $\mathrm{D}_{2}$ Resonance Line, C.R. Acad. Sci. Paris. Ser. B 286, 253 (1978).

[5] S. Liberman et al., Laser Optical Spectroscopy on Francium $\mathrm{D}_{2}$ Resonance Line, Phys. Rev. A 22, 2732 (1980).

[6] H. Yagoda, The Ultimate Lines of Element 87, Phys. Rev. 40, 1017 (1932).

[7] A. Coc et al., Hyperfine Structures and Isotope Shifts of ${ }^{207-213,220-228}$ Fr; Possible Evidence of Octupolar Deformation, Phys. Lett. 163B, 66 (1985).

[8] F. Touchard et al., Laser Spectroscopy of Francium Atoms, At. Masses Fund. Const. 7, 353 (1984).

[9] J Bauche et al., Accurate Wavenumbers of the First Resonance Doublet of Francium Atoms and Relativistic Contribution to Isotope Shifts in the Resonance Doublets of Francium and Caesium Atoms, J. Phys. B 19, L593 (1986).

[10] H. T. Duong et al., First Observation of the Blue Optical Lines of Francium, Europhys. Lett. 3, 175 (1987).

[11] S. V. Andreev, V. S. Letokhov, and V. I. Mishin, Laser Resonance Photoionization Spectroscopy of Rydberg Levels in Fr, Phys. Rev. Lett. 59, 1274 (1987).

[12] Y. A. Kudriavtsev and V.S. Letokhov, Laser Method of Highly Selective Detection of Rare Radioactive Isotopes through Multistep Photoionization of Accelerated Atoms, Appl. Phys. B 29, 219 (1982).

[13] C. Schulz et al., Resonance Ionization Spectroscopy on a Fast Atomic Ytterbium Beam, J. Phys. B 24, 4831 (1991).

[14] C. N. Davids et al., Proton Decay of an Intruder State in ${ }^{185} \mathrm{Bi}$, Phys. Rev. Lett. 76, 592 (1996).

[15] H. Kettunen et al., Investigations into the Alpha-Decay of ${ }^{195}$ At, Eur. Phys. J. A 16, 457 (2003).

[16] J. Uusitalo et al., $\alpha$ Decay Studies of Very Neutron-Deficient Francium and Radium Isotopes, Phys. Rev. C 71, 024306 (2005).

[17] U. Jakobsson et al., Recoil-Decay Tagging Study of ${ }^{205} \mathrm{Fr}$, Phys. Rev. C 85, 014309 (2012).
[18] M. Huyse, P. Decrock, P. Dendooven, G. Reusen, P. Van Duppen, and J. Wauters, Isomers in Three Doubly Odd Fr-At-Bi $\alpha$-Decay Chains, Phys. Rev. C 46, 1209 (1992).

[19] U. Jakobsson et al., Spectroscopy of the Proton Drip-Line Nucleus ${ }^{203}$ Fr, Phys. Rev. C 87, 054320 (2013).

[20] A. Voss, M. R. Pearson, J. Billowes, F. Buchinger, B. Cheal, J. E. Crawford, A. A. Kwiatkowski, C. D. P. Levy, and O. Shelbaya, First Use of High-Frequency Intensity Modulation of Narrow-Linewidth Laser Light and Its Application in Determination of ${ }^{206,205,204} \mathrm{Fr}$ Ground-State Properties, Phys. Rev. Lett. 111, 122501 (2013).

[21] V. N. Fedosseev, Y. Kudryavtsev, and V. I. Mishin, Resonance Laser Ionization of Atoms for Nuclear Physics, Phys. Scr. 85, 058104 (2012).

[22] L. Weissman et al., Magnetic Moments of ${ }^{68} \mathrm{Cu}^{g, m}$ and ${ }^{70} \mathrm{Cu}^{g, m_{1}, m_{2}}$ Nuclei Measured by In-Source Laser Spectroscopy, Phys. Rev. C 65, 024315 (2002).

[23] I. Stefanescu et al., Coulomb Excitation of ${ }^{68,70} \mathrm{Cu}$ : First Use of Postaccelerated Isomeric Beams, Phys. Rev. Lett. 98, 122701 (2007).

[24] J. Van Roosbroeck et al., Unambiguous Identification of Three $\beta$-Decaying Isomers in ${ }^{70} \mathrm{Cu}$, Phys. Rev. Lett. 92 , 112501 (2004).

[25] E. Kugler, The ISOLDE Facility, Hyperfine Interact. 129, 23 (2000).

[26] A. Jokinen, M. Lindroos, E. Molin, and M. Petersson, RFQ-Cooler for Low-Energy Radioactive Ions at ISOLDE, Nucl. Instrum. Methods Phys. Res., Sect. B 204, 86 (2003).

[27] E. Mané et al., An Ion Cooler-Buncher for High-Sensitivity Collinear Laser Spectroscopy at ISOLDE, Eur. Phys. J. A 42, 503 (2009).

[28] W. H. Haynes, Handbook of Chemistry and Physics (CRC, Boca Raton, FL, 2013), 94th ed.

[29] V. N. Fedosseev et al., Upgrade of the Resonance Ionization Laser Ion Source at ISOLDE On-line Isotope Separation Facility: New Lasers and New Ion Beams, Rev. Sci. Instrum. 83, 02 A903 (2012).

[30] S. Rothe, V. N. Fedosseev, T. Kron, B. A. Marsh, R. E. Rossel, and K. D. A. Wendt, Narrow Linewidth Operation of the RILIS Titanium: Sapphire Laser at ISOLDE/CERN, Nucl. Instrum. Methods Phys. Res., Sect. B 317, 561 (2013).

[31] R. E. Rossel, V. N. Fedosseev, B. A. Marsh, D. Richter, S. Rothe, and K. D. A. Wendt, Data Acquisition, Remote Control and Equipment Monitoring for ISOLDE RILIS, Nucl. Instrum. Methods Phys. Res., Sect. B 317, 557 (2013).

[32] K. M. Lynch, T. E. Cocolios, and M. M. Rajabali, Laser Assisted Decay Spectroscopy at the CRIS Beam Line at ISOLDE, Hyperfine Interact. 216, 95 (2013).

[33] M. M. Rajabali et al., A Dedicated Decay-Spectroscopy Station for the Collinear Resonance Ionization Experiment at ISOLDE, Nucl. Instrum. Methods Phys. Res., Sect. A 707, 35 (2013).

[34] P. Dendooven, Ph. D. thesis, KU Leuven, 1992.

[35] A. N. Andreyev et al., New Type of Asymmetric Fission in Proton-Rich Nuclei, Phys. Rev. Lett. 105, 252502 (2010).

[36] J. Elseviers et al., $\beta$-Delayed Fission of ${ }^{180} \mathrm{Tl}$, Phys. Rev. C 88, 044321 (2013).

[37] B. Lommel, W. Hartmann, B. Kindler, J. Klemm, and J. Steiner, Preparation of Self-Supporting Carbon Thin Films, Nucl. Instrum. Methods Phys. Res., Sect. A 480, 199 (2002). 
[38] W. Hennig, H. Tan, M. Walby, P. Grudberg, A. FalluLabruyere, W. K. Warburton, C. Vaman, K. Starosta, and D. Miller, Clock and Trigger Synchronization between Several Chassis of Digital Data Acquisition Modules, Nucl. Instrum. Methods Phys. Res., Sect. B 261, 1000 (2007).

[39] K. T. Flanagan et al., Collinear Resonance Ionization Spectroscopy of Neutron-Deficient Francium Isotopes, Phys. Rev. Lett. 111, 212501 (2013).

[40] I. Budinčević et al. (unpublished).

[41] T. E. Cocolios et al., The Collinear Resonance Ionization Spectroscopy (CRIS) Experimental Setup at CERNISOLDE, Nucl. Instrum. Methods Phys. Res., Sect. B 317, 565 (2013).

[42] K. M. Lynch, Ph. D. thesis, The University of Manchester, 2013.

[43] H. De Witte et al., Alpha-Decay of Neutron-Deficient ${ }^{200} \mathrm{Fr}$ and Heavier Neighbours, Eur. Phys. J. A 23, 243 (2004).

[44] S. Zhu and F. G. Kondev, Nuclear Data Sheets for $A=202$, Nucl. Data Sheets 109, 699 (2008).

[45] W. H. King, Comments on the Article "Peculiarities of the Isotope Shift in the Samarium Spectrum," J. Opt. Soc. Am. 53, 638 (1963).

[46] V. A. Dzuba, W. R. Johnson, and M. S. Safronova, Calculation of Isotope Shifts for Cesium and Francium, Phys. Rev. A 72, 022503 (2005).

[47] W. Lourens, Ph. D. thesis, Technische Hogeschool Delft, 1967.

[48] B. G. Ritchie, K. S. Toth, H. K. Carter, R. L. Mlekodaj, and E. H. Spejewski, Alpha-Decay Properties of ${ }^{205,206,207,208} \mathrm{Fr}$ : Identification of ${ }^{206} \mathrm{Fr}^{m}$, Phys. Rev. C 23, 2342 (1981).

[49] F. G. Kondev and S. Lalkovski, Nuclear Data Sheets for $A=207$, Nucl. Data Sheets 112, 707 (2011).

[50] B. Singh, D. Abriola, C. Baglin, V. Demetriou, T. Johnson, E. McCutchan, G. Mukherjee, S. Singh, A. Sonzogni, and J. Tuli, Nuclear Data Sheets for A $=211$, Nucl. Data Sheets 114, 661 (2013).

[51] E. Browne and J. K. Tuli, Nuclear Data Sheets for $A=220$, Nucl. Data Sheets 112, 1115 (2011).

[52] E. Gomez, S. Aubin, L. A. Orozco, G. D. Sprouse, E. Iskrenova-Tchoukova, and M. S. Safronova, Nuclear Magnetic Moment of ${ }^{210} \mathrm{Fr}$ : A Combined Theoretical and Experimental Approach, Phys. Rev. Lett. 100, 172502 (2008).

[53] K. M. Lynch et al., CRIS: A New Method in Isomeric Beam Production, Eur. Phys. J. Web Conf. 63, 01007 (2013).

[54] C. Ekström, L. Robertsson, and A. Rosén, Nuclear and Electronic g-Factors of ${ }^{211} \mathrm{Fr}$, Nuclear Ground-State Spin of ${ }^{207} \mathrm{Fr}$ and the Nuclear Single-Particle Structure in the Range ${ }^{207-228}$ Fr, Phys. Scr. 34, 624 (1986).

[55] H. H. Stroke, R. J. Blin-Stoyle, and V. Jaccarino, Configuration Mixing and the Effects of Distributed Nuclear Magnetization on Hyperfine Structure in Odd-A Nuclei, Phys. Rev. 123, 1326 (1961).

[56] M. Anselment, W. Faubel, S. Göring, A. Hanser, G. Meisel, H. Rebel, and G. Schatz, The Odd-Even Staggering of the Nuclear Charge Radii of $\mathrm{Pb}$ Isotopes, Nucl. Phys. A451, 471 (1986).
[57] W. D. Myers and K.-H. Schmidt, An Update on DropletModel Charge Distributions, Nucl. Phys. A410, 61 (1983).

[58] S. Raman, C. W. Nestor, Jr., and P. Tikkanen, Transition Probability from the Ground to the First-Excited $2^{+}$State of Even-Even Nuclides, At. Data Nucl. Data Tables 78, 1 (2001).

[59] H. De Witte et al., Nuclear Charge Radii of NeutronDeficient Lead Isotopes Beyond $N=104$ Midshell Investigated by In-Source Laser Spectroscopy, Phys. Rev. Lett. 98, 112502 (2007).

[60] W. Borchers, R. Neugart, E. W. Otten, H. T. Duong, G. Ulm, and K. Wendt, Hyperfine Structure and Isotope Shift Investigations in ${ }^{202-222} \mathrm{Rn}$ for the Study of Nuclear Structure beyond $Z=82$, Hyperfine Interact. 34, 25 (1987).

[61] T. Bastug, B. Fricke, M. Finkbeiner, and W. R. Johnson, The Magnetic Moment of ${ }^{209} \mathrm{Bi}-A$ Molecular Determination of the Diamagnetic Shielding, Z. Phys. D 37, 281 (1996).

[62] R. Neugart, H. H. Stroke, S. A. Ahmad, H. T. Duong, H. L. Ravn, and K. Wendt, Nuclear Magnetic Moment of ${ }^{207} \mathrm{Tl}$, Phys. Rev. Lett. 55, 1559 (1985).

[63] N. Stone, Table of Nuclear Magnetic Dipole and Electric Quadrupole Moments (Nuclear Data Services, International Atomic Energy Agency, Vienna, Austria, 2011).

[64] G. Neyens, Nuclear Magnetic and Quadrupole Moments for Nuclear Structure Research on Exotic Nuclei, Rep. Prog. Phys. 66, 633 (2003).

[65] A. E. Barzakh, L. K. Batist, D. V. Fedorov, V. S. Ivanov, K. A. Mezilev, P. L. Molkanov, F. V. Moroz, S. Y. Orlov, V. N. Panteleev, and Y.M. Volkov, Hyperfine Structure Anomaly and Magnetic Moments of Neutron Deficient $T l$ Isomers with $I=9 / 2$, Phys. Rev. C 86, 014311 (2012).

[66] J. Wouters, N. Severijns, J. Vanhaverbeke, and L. Vanneste, Magnetic Moments of Po Isotopes and the Quenching of Nuclear Magnetism in the ${ }^{208} \mathrm{~Pb}$ Region, J. Phys. G 17, 1673 (1991).

[67] S. A. Ahmad, W. Klempt, R. Neugart, E. W. Otten, K. Wendt, and C. Ekström, Determination of Nuclear Spins and Moments in a Series of Radium Isotopes, Phys. Lett. 133B, 47 (1983).

[68] P. Van Duppen, P. Decrock, P. Dendooven, M. Huyse, G. Reusen, and J. Wauters, Intruder States in Odd-Odd $\mathrm{Tl}$ Nuclei Populated in the $\alpha$-Decay of Odd-Odd Bi Isotopes, Nucl. Phys. A529, 268 (1991).

[69] F. G. Kondev, Nuclear Data Sheets for $A=206$, Nucl. Data Sheets 109, 1527 (2008).

[70] C. J. Chiara and F. G. Kondev, Nuclear Data Sheets for $A=204$, Nucl. Data Sheets 111, 141 (2010).

[71] M. J. Martin, Nuclear Data Sheets for $A=208$, Nucl. Data Sheets 108, 1583 (2007).

[72] E. Browne, Nuclear Data Sheets for $A=212$, Nucl. Data Sheets 104, 427 (2005).

[73] K. M. Lynch et al., Laser Assisted Decay Spectroscopy at the CRIS Beam Line at ISOLDE, J. Phys. Conf. Ser. 381, 012128 (2012). 